\title{
Nomenclatural review of Acalypha (Euphorbiaceae) of the Western Indian Ocean Region (Madagascar, the Comoros Archipelago, the Mascarene Islands and the Seychelles Archipelago)
}

\author{
Iris Montero Muñoz', José María Cardiel', Geoffrey A. Levinn ${ }^{2,3}$ \\ I Departamento de Biologia, Facultad de Ciencias, Universidad Autónoma de Madrid, Ciudad Universitaria \\ de Cantoblanco, 28049, Madrid, Spain 2 Illinois Natural History Survey, Prairie Research Institute, University \\ of Illinois, 1816 S Oak St., Champaign, IL 61820, USA 3 Canadian Museum of Nature, P.O. Box 3443, \\ Station D, Ottawa, ON K1P 6P4, Canada \\ Corresponding author: Iris Montero Muñoz (iris.montero@uam.es)
}

Academic editor: R. Riina | Received 14 June 2018 | Accepted 7 August 2018 | Published 10 September 2018

Citation: Montero Muñoz I, Cardiel JM, Levin GA (2018) Nomenclatural review of Acalypha (Euphorbiaceae) of the Western Indian Ocean Region (Madagascar, the Comoros Archipelago, the Mascarene Islands and the Seychelles Archipelago). PhytoKeys 108: 85-116. https://doi.org/10.3897/phytokeys.108.27284

\begin{abstract}
This work presents a critical nomenclatural review of the Acalypha species of the Western Indian Ocean Region (Madagascar, the Comoros Archipelago, the Mascarene Islands and the Seychelles Archipelago). This is the first treatment of Acalypha of Madagascar since Leandri's monograph in 1942. A total of 151 scientific names related to Acalypha from this region are treated. We recognise 35 species (28 native and seven introduced), treat 93 names as synonyms ( 28 of them for the first time) and identify three as doubtful or excluded names. We designate lectotypes for 41 names, make two new combinations and propose one new name.
\end{abstract}

\section{Keywords}

Acalypha, Comoros, Euphorbiaceae, Madagascar, Mascarenes, nomenclature, Seychelles, synonymy

Copyright Iris Montero Muñoz et al. This is an open access article distributed under the terms of the Creative Commons Attribution License (CC BY 4.0), which permits unrestricted use, distribution, and reproduction in any medium, provided the original author and source are credited. 


\section{Introduction}

This work is part of the taxonomic and biogeographic revision of Acalypha (Euphorbiaceae) in the Western Indian Ocean Region (WIOR). According to the Taxonomic Databases Working Group standards (Brummit 2001), this region includes Madagascar, the Mascarene Islands (Mauritius and the French Department of Réunion), the Comoros Islands (Union of the Comoros and the French Department of Mayotte) and the Seychelles Archipelago. There are no species of Acalypha known from any of the smaller Scattered Islands of the Western Indian Ocean.

The first two species of Madagascan Acalypha (A. fliformis and A. venosa) were described by Poiret (1804) in Lamarck's encyclopaedia. More than half a century later, Baillon (1861) published Euphorbiacées Africaines, Afrique Orientale (Bourbon, Maurice, Madagascar, Zanguebar etc.), where 21 species of Acalypha are cited, of which 19 are from the study area. Baker $(1883,1884), 20$ years later, described four new species. In 1891, Baillon, in his contribution to Grandidier's Histoire Physique, Naturelle et Politique de Madagascar, provided 10 excellent plates of Acalypha species from Madagascar, five of which are cited for the first time (Baillon 1891). These plates are not accompanied by descriptions or any additional information. Soon thereafter, Baillon (1892, 1895a, 1895b) published in several chapters his Liste des Plantes de Madagascar citing 32 Acalypha species, six of which were newly described.

In the treatment of Acalypha for Engler's Das Pfanzenreich, Pax and Hoffmann (1924) included 31 species from WIOR. In this work, Acalypha was formally divided into three subgenera, "Euacalypha", Androcephala and Linostachys; the first two occur in WIOR. The most recent work treating Madagascan Acalypha (Leandri 1942) is now almost 80 years old. Leandri's work included complete information about 22 species and 14 varieties of Acalypha, of which eight species and six varieties were described for the first time.

Only three floristic works included Acalypha species from the other islands of the Western Indian Ocean region. Baker (1877) cited five species from Mauritius and the Seychelles and de Cordemoy (1895) treated five species from Réunion. Most recently, Coode (1982) included five species, three subspecies and seven varieties from the Mascarene Islands.

While undertaking this nomenclatural review, we found 144 names related to Acalypha from the study area, many of which involved serious problems of taxonomic identity. All of these names have been evaluated in this work and our interpretation of their current taxonomic status and distribution is presented in Tables 1 and 2. Our aim is to lay the foundation for a complete taxonomic and biogeographical revision of Acalypha in the WIOR, a region of particular relevance to the origin and evolution of this genus. Preliminary results of Acalypha molecular phylogeny (Levin et al. 2005) suggest that the genus first appeared in Africa, where the highest morphological diversity within the genus is found. We share the same purpose as the recently published WIOR nomenclator of Croton (Berry et al. 2017), the second largest genus of the Euphorbiaceae, preceded by Euphorbia (Horn et al. 2012) and followed by Acalypha. 


\section{Materials and methods}

We conducted a thorough bibliographical review and consulted the following herbaria for the Western Indian Ocean Acalypha types: B, BM, BREM, BRNU, C, CAS, G, GB, GDC, GH, JE, K, M, MA, MAU, MO, MPU, NY, P, S, US, TAN, TUB and W (acronyms according to Thiers 2018). Scanned images of types from these and other herbaria, available on JSTOR Global Plants (http://plants.jstor.org/), were also consulted.

The accepted species are cited in alphabetical order and all known synonyms are included, except for the introduced species. We include the notation "syn. nov." whenever we newly treat a taxon as a synonym. We provide details of the type collection(s) for each name. Lectotypes are designated after a careful review of the relevant original literature and examination of the nomenclatural types.

Five of the Acalypha treated names were illustrated first, without description, in Baillon's Histoire Naturelle des Plantes (Baillon 1891). These are A. diminuta, A. humblotiana, A. leptomyura, A. madreporica and A. polynema. Except A. humblotiana, we consider each of the plates provided, which include extensive vegetative and reproductive morphological details, as the holotypes of the associated names, according to art. 38.8 of the ICN (McNeill et al. 2012).

When two or more syntypes were cited in the protologue, a lectotype is designated, using the best preserved specimen or the one most consistent with the protologue. The remaining syntypes are also cited.

The type locality information is taken mainly from the type specimen labels. We add additional information such as the province name of Madagascar or a modern or accepted spelling of a place name. If the locality is ambiguous or imprecise, we keep the literal citation (in quotation marks) as it appears in the protologue. Barcode numbers of type specimens are cited when available.

Under the "Distribution" section, we list the general distribution of each species in Africa (following Brummit 2001), if they occur there and in the WIOR region. We include the Madagascar provinces or island names within the studied archipelagos where they occur. This information should be taken as preliminary until a more complete study of the genus, in progress, is completed.

In the references section, we only include references which cite Acalypha in the WIOR region.

All information gathered as part of this work, including the complete listing of studied specimens, is available online at the regularly updated Acalypha Taxonomic Information System website, www.acalypha.es (Cardiel et al. 2018).

\section{Results and discussion}

In the present work, we record 151 published scientific names related to Acalypha of the WIOR (Table 1). Of these, 35 are accepted names, 93 are considered synonyms and 
Table I. Taxa included in Acalypha of the Western Indian Ocean Region. Accepted names in bold.

\begin{tabular}{|c|c|}
\hline Published names & Accepted names in this paper \\
\hline A. acuminata Vahl ex Baill. nom. illeg. & A. burmanii I.Montero \& Cardiel nom. nov. \\
\hline A. aldabrica Pax \& K.Hoffm. & A. claoxyloides Hutch. \\
\hline A. amentacea Roxb. subsp. wilkesiana (Müll.Arg.) Fosberg & A. wilkesiana Müll.Arg. \\
\hline A. andringitrensis Leandri & A. radula Baker \\
\hline A. arborea Comm. in Poir. nom. nud. & A. filiformis Poir. \\
\hline A. aspretorum Leandri nom. nud. & A. medibracteata Radcl.-Sm. \& Govaerts \\
\hline \multicolumn{2}{|l|}{ A. bailloniana Müll.Arg. } \\
\hline A. bakeriana Baill. & A. emirnensis Baill. \\
\hline A. baronii Baker & A. emirnensis Baill. \\
\hline \multicolumn{2}{|l|}{ A. boinensis Leandri } \\
\hline A. buchenavii Müll.Arg. & A. spachiana Baill. \\
\hline \multicolumn{2}{|l|}{ A. burmanii I.Montero \& Cardiel nom. nov. } \\
\hline \multicolumn{2}{|l|}{ A. chibomboa Baill. } \\
\hline \multicolumn{2}{|l|}{ A. claoxyloides Hutch. } \\
\hline A. cloiselana M. Denis in Leandri nom. nud. & A. gracilipes Baill. \\
\hline A. codonocalyx Baill. & A. chibomboa Baill. \\
\hline A. colorata (Poir.) Spreng. & A. integrifolia Willd. \\
\hline A. commersoniana Baill. nom. nud. & A. integrifolia Willd. \\
\hline A. commersoniana Baill. ex Müll.Arg. & A. integrifolia Willd. \\
\hline A. commersoniana var. acutifolia Müll.Arg. & A. integrifolia Willd. \\
\hline A. commersoniana var. acutifolia f. concolor Müll.Arg. & A. integrifolia Willd. \\
\hline A. commersoniana var. acutifolia f. purpurea Müll.Arg. & A. integrifolia Willd. \\
\hline A. commersoniana var. acutifolia f. purpureo-marginata Müll.Arg. & A. integrifolia Willd. \\
\hline A. commersoniana var. brevifolia Baill. ex Müll.Arg. & A. integrifolia Willd. \\
\hline A. commersoniana var. concolor Baill. nom. nud. & A. integrifolia Willd. \\
\hline A. commersoniana var. discolor Baill. nom. nud. & A. integrifolia Willd. \\
\hline A. commersoniana var. gracilipes (Baill.) Müll.Arg. & A. gracilipes Baill. \\
\hline A. commersoniana var. longifolia Müll.Arg. & A. integrifolia Willd. \\
\hline A. commersoniana var. obtusifolia Müll.Arg. & A. integrifolia Willd. \\
\hline A. commersoniana var. obtusifolia f. colorata (Poir.) Müll.Arg. & A. integrifolia Willd. \\
\hline A. commersoniana var. obtusifolia $\mathrm{f}$. discolor Müll.Arg. & A. integrifolia Willd. \\
\hline A. commersoniana var. obtusifolia f. unicolor Müll.Arg. & A. integrifolia Willd. \\
\hline A. commersoniana var. parvifolia Baill. nom. nud. & A. integrifolia Willd. \\
\hline A. commersoniana var. parvifolia Baill. ex Müll.Arg. & A. integrifolia Willd. \\
\hline A. commersonii Baill. nom. nud. & A. gracilipes Baill. \\
\hline A. comorensis $\operatorname{Pax}$ & A. chibomboa Baill. \\
\hline A. crenata var. glandulosa Müll.Arg. & $\begin{array}{l}\text { A. lanceolata var. glandulosa (Müll.Arg.) } \\
\text { Radcl.-Sm. }\end{array}$ \\
\hline \multicolumn{2}{|l|}{ A. decaryana Leandri } \\
\hline \multicolumn{2}{|l|}{ A. diminuta Baill. } \\
\hline A. discolor Bojer nom. nud. & A. integrifolia Willd. \\
\hline \multicolumn{2}{|l|}{ A. emirnensis Baill. } \\
\hline A. emirnensis var. bara Leandri & A. emirnensis Baill. \\
\hline
\end{tabular}




\begin{tabular}{|c|c|}
\hline Published names & Accepted names in this paper \\
\hline A. emirnensis var. jabohaziana Leandri & A. emirnensis Baill. \\
\hline \multicolumn{2}{|l|}{ A. fasciculata Müll.Arg. } \\
\hline A. fasciculata var. humbertiana Leandri & A. fasciculata Müll.Arg. \\
\hline A. fasciculata var. lyallii (Baker) Leandri & A. fasciculata Müll.Arg. \\
\hline \multicolumn{2}{|l|}{ A. fliformis Poir. } \\
\hline A. fliformis var. arborea Poir. & A. filiformis Poir. \\
\hline A. fliformis var. goudotiana (Baill.) Govaerts & A. urophylla Boivin ex Baill. \\
\hline A. filiformis var. ovalifolia (Baill.) Govaerts & A. ovalifolia Baill. \\
\hline A. filiformis var. pervilleana (Baill.) Govaerts & A. paxii Aug.DC. \\
\hline A. filiformis var. urophylla (Boivin ex Baill.) Govaerts & A. urophylla Boivin ex Baill. \\
\hline A. filiformis var. urophylloides (Pax \& K.Hoffm.) Govaerts & A.paxii Aug.DC. \\
\hline \multicolumn{2}{|l|}{ A. fimbriata Schumach. \& Thonn. } \\
\hline A. fryeri Hutch. & A. claoxyloides Hutch. \\
\hline A. gagnepainii Leandri nom. illeg. & A. medibracteata Radcl.-Sm. \& Govaerts \\
\hline A. gagnepainii var. calcicola Leandri & A. medibracteata Radcl.-Sm. \& Govaerts \\
\hline A. goudotiana Baill. & A. urophylla Boivin ex Baill. \\
\hline \multicolumn{2}{|l|}{ A. gracilipes Baill. } \\
\hline A. hildebrandtii Baill. & A. radula Baker \\
\hline \multicolumn{2}{|l|}{ A. hispida Burm.f. } \\
\hline A. hologyna Baker & A. emirnensis Baill. \\
\hline \multicolumn{2}{|l|}{ A. humbertii Leandri } \\
\hline A. humblotiana Baill. & A. paxii Aug.DC. \\
\hline \multicolumn{2}{|l|}{ A. indica $\mathrm{L}$. } \\
\hline A. indica var. bailloniana (Müll.Arg.) Hutch. & A. bailloniana Müll.Arg. \\
\hline \multicolumn{2}{|l|}{ A. integrifolia Willd. } \\
\hline A. integrifolia subsp. marginata (Poir.) Coode & A. marginata (Poir.) Spreng. \\
\hline A. integrifolia subsp. marginata var. crateriana Coode & A. marginata (Poir.) Spreng. \\
\hline A. integrifolia subsp. marginata var. saltuum Coode & A. marginata (Poir.) Spreng. \\
\hline A. integrifolia subsp. panduriformis Coode & A. marginata (Poir.) Spreng. \\
\hline A. integrifolia var. colorata (Poir.) Pax \& K.Hoffm. & A. integrifolia Willd. \\
\hline A. integrifolia var. concolor (Müll.Arg.) Pax \& K.Hoffm. & A. integrifolia Willd. \\
\hline A. integrifolia var. gracilipes (Baill.) Pax \& K.Hoffm. & A. gracilipes Baill. \\
\hline A. integrifolia var. longifolia (Müll.Arg.) Coode & A. integrifolia Willd. \\
\hline $\begin{array}{l}\text { A. integrifolia var. parvifolia (Baill. ex Müll.Arg.) Pax \& } \\
\text { K.Hoffm. }\end{array}$ & A. integrifolia Willd. \\
\hline A. juliflora Pax & A. rottleroides Baill. \\
\hline \multicolumn{2}{|l|}{ A. lamiana (Leandri) I.Montero \& Cardiel comb. nov. } \\
\hline \multicolumn{2}{|l|}{ A. lanceolata var. glandulosa (Müll.Arg.) Radcl.-Sm. } \\
\hline A. Lantanaefolia Bojer nom. nud. & A. filiformis Poir. \\
\hline \multicolumn{2}{|l|}{ A. leonii Baill. } \\
\hline A. leonii var. perrierana Leandri & A. leonii Baill. \\
\hline \multicolumn{2}{|l|}{ A. lepidopagensis Leandri } \\
\hline \multicolumn{2}{|l|}{ A. leptomyura Baill. } \\
\hline A. linearifolia Leandri & \\
\hline
\end{tabular}




\begin{tabular}{|c|c|}
\hline Published names & Accepted names in this paper \\
\hline A. lyallii Baker & A. fasciculata Müll.Arg. \\
\hline A. madagascariensis Pax \& K.Hoffm. & A. fasciculata Müll.Arg. \\
\hline A. madreporica Baill. & A. fasciculata Müll.Arg. \\
\hline \multicolumn{2}{|l|}{ A. marginata (Poir.) Spreng. } \\
\hline \multicolumn{2}{|l|}{ A. medibracteata Radcl.-Sm. \& Govaerts } \\
\hline A. medibracteata var. calcicola (Leandri) Radcl.-Sm. \& Govaerts & A. medibracteata Radcl.-Sm. \& Govaerts \\
\hline A. meiodonta Baill. & A. paxii Aug.DC. \\
\hline A. menabeana Leandri nom. nud. & A. medibracteata Radcl.-Sm. \& Govaerts \\
\hline \multicolumn{2}{|l|}{ A. menavody (Leandri) I.Montero \& Cardiel comb. nov. } \\
\hline \multicolumn{2}{|l|}{ A. neptunica Müll.Arg. } \\
\hline A. ovalifolia Baill. & A. richardiana Baill. \\
\hline \multicolumn{2}{|l|}{ A.paxii Aug.DC. } \\
\hline \multicolumn{2}{|l|}{ A. perrieri Leandri } \\
\hline A. pervilleana Baill. & A. paxii Aug.DC. \\
\hline \multicolumn{2}{|l|}{ A. poiretii Spreng. } \\
\hline A. polynema Baill. & A. rottleroides Baill. \\
\hline A. radula Baker & A. radula Baker \\
\hline A. reticulata (Poir.) Müll.Arg. & A. fliformis Poir. \\
\hline A. reticulata var. arborea (Poir.) Müll.Arg. & A. filiformis Poir. \\
\hline A. reticulata var. cloiselana Leandri & A. gracilipes Baill. \\
\hline A. reticulata var. goudotiana (Baill.) Müll.Arg. & A. urophylla Boivin ex Baill. \\
\hline A. reticulata var. longifolia Müll.Arg. & A. integrifolia Willd. \\
\hline A. reticulata var. longifolia f. aberrans Müll.Arg. & A. integrifolia Willd. \\
\hline A. reticulata var. meiodonta (Baill.) Leandri & A. paxii Aug.DC. \\
\hline A. reticulata var. meiodonta f. andronea Leandri & A. paxii Aug.DC. \\
\hline A. reticulata var. ovalifolia (Baill.) Müll.Arg. & A. ovalifolia Baill. \\
\hline A. reticulata var. pervilleana (Baill.) Müll.Arg. & A. paxii Aug.DC. \\
\hline A. reticulata var. urophylla (Boivin ex Baill.) Müll.Arg. & A. urophylla Boivin ex Baill. \\
\hline A. reticulata var. urophylla f. glabrescens Leandri & A. urophylla Boivin ex Baill. \\
\hline A. reticulata var. urophylla f. humblotiana (Baill.) Leandri & A. paxii Aug.DC. \\
\hline A. reticulata var. urophylla f. Lamiana Leandri & $\begin{array}{l}\text { A. lamiana (Leandri) I.Montero \& Cardiel } \\
\text { comb. nov. }\end{array}$ \\
\hline A. reticulata var. urophylla f. longa Leandri & A. urophylla Boivin ex Baill. \\
\hline A. reticulata var. urophylla f. meeusei Leandri & A. urophylla Boivin ex Baill. \\
\hline A. reticulata var. urophylla f. typique Leandri nom. inval. & A. urophylla Boivin ex Baill. \\
\hline A. reticulata var. urophylla f. vohitrae Leandri & A. urophylla Boivin ex Baill. \\
\hline A. reticulata var. urophylloides Pax \& K.Hoffm. & A. paxii Aug.DC. \\
\hline \multicolumn{2}{|l|}{ A. richardiana Baill. } \\
\hline \multicolumn{2}{|l|}{ A. rottleroides Baill. } \\
\hline A. salviifolia Baill. nom. nud. & A. radula Baker \\
\hline \multicolumn{2}{|l|}{ A. spachiana Baill. } \\
\hline A. spachiana var. acutifolia Baill. & A. spachiana Baill. \\
\hline A. spachiana var. latifolia Baill. & A. spachiana Baill. \\
\hline A. spachiana var. minor Baill. & A. spachiana Baill. \\
\hline A. spiciflora Burm.f. & Claoxylon spiciflorum (Burm.f.) A.Juss. \\
\hline A. spiciflora var. menavody Leandri & $\begin{array}{l}\text { A. menavody (Leandri) I.Montero \& Cardiel } \\
\text { comb. nov. }\end{array}$ \\
\hline
\end{tabular}




\begin{tabular}{|c|c|}
\hline Published names & Accepted names in this paper \\
\hline A. squarrosa $\operatorname{Pax}$ & A. spachiana Baill. \\
\hline \multicolumn{2}{|l|}{ A. urophylla Boivin ex Baill. } \\
\hline A. urophylla Pax nom. illeg. & A. paxii Aug.DC. \\
\hline A. venosa Poir. & Leptonema venosum (Poir.) A.Juss. \\
\hline \multicolumn{2}{|l|}{ A. vulneraria Baill. } \\
\hline \multicolumn{2}{|l|}{ A. wilkesiana Müll.Arg. } \\
\hline Caturus sessilis Pet.Thou. in Baill. nom. nud. & A. integrifolia Willd. \\
\hline Ricinocarpus baillonianus (Müll.Arg) Kuntze & A. bailloniana Müll.Arg. \\
\hline Ricinocarpus hispidus (Brum.f.) Kuntze & A. hispida Brum.f. \\
\hline Ricinocarpus poiretii (Spreng.) Kuntze & A. poiretii Spreng. \\
\hline Ricinocarpus wilkesianus (Müll.Arg.) Fosberg & A. wilkesiana Müll.Arg. \\
\hline Tragia colorata Poir. & A. integrifolia Willd. \\
\hline Tragia filiformis Poir. & A. burmanii I.Monero \& Cardiel nom. nov. \\
\hline Tragia fruticosa Commers. in Baill. nom. nud. & A. integrifolia Willd. \\
\hline Tragia lobata Wall. nom. nud. & A. integrifolia Willd. \\
\hline Tragia macrophylla Wall. nom. nud. & A. integrifolia Willd. \\
\hline Tragia marginata Poir. & A. integrifolia Willd. \\
\hline Tragia obtusata Vahl. in Baill. nom. nud. & A. integrifolia Willd. \\
\hline Tragia reticulata Poir. & A. filiformis Poir. \\
\hline Tragia salviifolia Bojer in Baill. nom. nud. & A. radula Baker \\
\hline Tragia saxatilis Bojer in Pax \& K.Hoffm. nom. nud. & A. spachiana Baill. \\
\hline
\end{tabular}

three are doubtful or excluded (A. neptunica Müll.Arg., A. spiciflora Burm.f. and A. venosa Poir.). We also include 19 nomina nuda found in literature and one nomen invalidum. Type specimens are indicated for all valid names and we have designated 41 lectotypes.

In Madagascar, we accept 28 species, 23 native (of which 20 are endemic) and five introduced (Table 2). Leandri (1942) recognised 22 species of Acalypha from Madagascar, of which we accept 19 (including $A$. gagnepainii under its correct name, $A$. medibracteata). We consider $A$. andringitrensis to be a synonym of $A$. radula. Our concept of what Leandri called $A$. reticulata is narrower than his; we consider $A$. reticulata s.s. (under its correct name, $A$. filiformis) to be endemic to the Mascarines and treat the Madagascan material Leandri assigned to this species as four distinct species ( $A$. gracilipes, A. lamiana, A. paxii and A. urophylla). We exclude the name Acalypha spiciflora (accepted by Leandri), because it is not an Acalypha. We report two species, $A$. fimbriata and $A$. lanceolata, not previously cited for Madagascar. We also treat the name A. madagascariensis, previously excluded by Leandri, as a synonym of $A$. fasciculata. We anticipate the description of at least 2 more new species from Madagascar based on the material we now have on hand.

In the Comoros Archipelago, there are seven species of Acalypha, four native (one endemic) and three introduced, one of which, A. bailloniana, is first reported here. There are seven species on the Mascarene Islands, three native (all endemic) and four introduced. There are four species on the Seychelles, one endemic and three introduced. 
Table 2. Synoptic table of the species distribution of Acalypha of the Western Indian Ocean Region (E: endemic, N: native, I: introduced).

\begin{tabular}{|c|c|c|c|c|}
\hline Species & Madagascar & Comoros & Mascarenes & Seychelles \\
\hline A. bailloniana & & I & & \\
\hline A. boinensis & $\mathrm{E}$ & & & \\
\hline A. burmanii & $\mathrm{E}$ & & & \\
\hline A. chibomboa & $\mathrm{N}$ & $\mathrm{N}$ & & \\
\hline A. claoxyloides & & & & $\mathrm{E}$ \\
\hline A. decaryana & $\mathrm{E}$ & & & \\
\hline A. diminuta & E & & & \\
\hline A. emirnensis & $\mathrm{E}$ & & & \\
\hline A. fasciculata & E & & & \\
\hline A. filiformis & & & $\mathrm{E}$ & \\
\hline A. fimbriata & I & & & \\
\hline A. gracilipes & $\mathrm{E}$ & & & \\
\hline A. humbertii & E & & & \\
\hline A. indica & I & I & I & I \\
\hline A. integrifolia & & & E & \\
\hline A. lamiana & $\mathrm{E}$ & & & \\
\hline A. lanceolata var. glandulosa & I & & I & \\
\hline A. leonii & $\mathrm{E}$ & & & \\
\hline A. lepidopagensis & $\mathrm{E}$ & & & \\
\hline A. leptomyura & E & & & \\
\hline A. linearifolia & $E$ & & & \\
\hline A. marginata & & & $\mathrm{E}$ & \\
\hline A. medibracteata & $\mathrm{E}$ & & & \\
\hline A. menavody & E & & & \\
\hline A. paxii & $\mathrm{N}$ & $\mathrm{N}$ & & \\
\hline A. perrieri & $\mathrm{E}$ & & & \\
\hline A. radula & $\mathrm{E}$ & & & \\
\hline A. richardiana & & $\mathrm{E}$ & & \\
\hline A. rottleroides & $\mathrm{E}$ & & & \\
\hline A. spachiana & $\mathrm{E}$ & & & \\
\hline A. urophylla & $\mathrm{N}$ & $\mathrm{N}$ & & \\
\hline A. vulneraria & $\mathrm{E}$ & & & \\
\hline A. hispida & I & I & I & I \\
\hline A. poiretii & & & I & \\
\hline A. wilkesiana & I & & & I \\
\hline Native & 23 & 4 & 3 & 1 \\
\hline Endemic & 20 & 1 & 3 & 1 \\
\hline Introduced & 5 & 3 & 4 & 3 \\
\hline Total & 28 & 7 & 7 & 4 \\
\hline
\end{tabular}


Nomenclatural synopsis of Malagasy, Comoros, Mascarene and Seychelles Acalypha

\section{Acalypha bailloniana Müll.Arg., Linnaea 34: 44. 1865.}

Ricinocarpus baillonianus (Müll.Arg.) Kuntze, Revis. Gen. Pl. 2: 617. 1891.

Acalypha indica var. bailloniana (Müll.Arg.) Hutch., Fl. Trop. Afr. 6(1): 904. 1912.

Type. Tanzania: Zanzibar: s.l., 1848, L. H. Boivin s.n. (holotype: P [P04809900]!).

Distribution. East Tropical Africa. Comoros Archipelago (Anjouan).

Notes. Acalypha bailloniana was considered as a subspecies of $A$. indica L. by Hutchinson (1913) and this treatment was followed in the subsequent floristic works. Radcliffe-Smith $(1987,1996)$ treated $A$. bailloniana as a synonym of $A$. indica. We consider that $A$. bailloniana is a distinct species which can be distinguished from $A$. indica by its dentante bracts with a prominent central tooth and with glandular hairs (vs. subentire bracts without prominent central tooth and without glandular hairs, in A. indica). A. bailloniana is distributed in the east coast of tropical Africa and cited for the WIOR region for the first time, where it is probably introduced.

\section{Acalypha boinensis Leandri, Notul. Syst. (Paris) 10: 268. 1942.}

Type. Madagascar. Prov. Mahajanga: Ambongo et Boïna, bassin moyen du Bemarivo, 200 m, Nov 1919, H. Perrier de la Bâthie 9823 (lectotype, designated here: P [P00508582]!).

Syntypes. Madagascar. Prov. Mahajanga: Maintirano, s.d., R. Decary 8216 P [P00508588]!; Madagascar. Prov. Mahajanga: Maromandia (Andranosamontana), s.d., R. Decary 1045 P [P00508585]!; Madagascar. Prov. Mahajanga: Morovoay, s.d., H. Humbert \& H. Perrier de la Bâthie 2350 P [P00508570!, P00508571!, P00508572!, P00508573!, P00508574!]; Madagascar. Prov. Mahajanga: Ambongo et Bö̈na, 300 m, s.d., H. Perrier de la Bâthie 9546 P [P00508576]!, 9551 P [P00508577]!; MadaGascar. Prov. Toliara: Forêt de Besomaty, entre le Fiherena et l'Isahaina (Mangoky), 750-800 m, s.d., H. Humbert 11234 P [P00508575]!; MadagasCar: s.l., s.d., Baron 5393 P [P00508586]!, 5450 [n.v.].

Distribution. Madagascar (Antsiranana, Fianarantsoa, Mahajanga, Toliara)

References. Govaerts et al. (2000: 51).

\section{Acalypha burmanii I.Montero \& Cardiel. nom. nov.} urn:lsid:ipni.org:names:60477001-2

Tragia filiformis Poir., Encycl. 7: 727. 1806. Acalypha acuminata Vahl ex. Baill. Adansonia 1: 267. 1861 nom. illeg., non. A. acuminata Benth. (1854).

Type. MadagasCaR?: s.l., s.d., Herb. de Lamarck. s.n. (holotype: P-LAM [P00367371]!). 
Distribution. MADAGASCAR (Antsiranana).

References. Müller Argoviensis (1865: 40) as A. spiciflora Burm.f.; Müller Argoviensis (1866: 867) as A. spiciflora; Baillon (1891: tab. 188) as A. acuminata Vahl. ex Baill.; Baillon (1892: 1004) as A. acuminata; Heckel (1903: 198) as A. acuminata; Palacký (1907: 24) as A. acuminata; Pax and Hoffmann (1924: 137) as A. spiciflora; Leandri (1942: 269) as A. spiciflora.

Notes. Acalypha burmanii is proposed as a new name for Tragia filiformis Poir. We cannot combine T. filiformis under Acalypha because it is blocked by A. filiformis Poir., nor can we use the illegitimate name $A$. acuminata Baill. See also comments under the excluded species $A$. spiciflora.

\section{Acalypha chibomboa Baill., Adansonia 1: 269. 1861.}

Type. Comoros, Anjouan, 1850, L. H. Boivin s.n. (holotype: P [P00196274]!).

Acalypha codonocalyx Baill., Adansonia 1: 271. 1861. Type: Comoros: Mohéli, "Ile Mohilla”, s.d., M. Richard 286 (lectotype, designated here: P [P00196282]!; isolectotype: P [P00196283]!).

Acalypha comorensis Pax, Bot. Jahrb. Syst. 19(1): 95. 1894. Type: Comoros, Anjouan, "Johanna", Jun-Aug 1875, J. M. Hildebrandt 1662 (holotype $\mathrm{B}$ or WRSL, presumably destroyed; lectotype, designated here: BREM [BREM0001792]!; isolectotypes: K [K000186524!, K000186525!], L [L0241274]!, P [P00196280]!, W [W0004243]!) Syntype: Comoros, Grande Comore, 1886. C. W. Schmidt 192 [n.v.].

Distribution. Madagascar (Antsiranana, Antananarivo), Comoros ArChipelago (Grande Comore, Anjouan, Mohéli, Mayotte).

References. Baillon (1891: Pl. 192 as A. codonocalyx); Palacký (1907: 24); Voeltzkow (1917: 447); Pax and Hoffmann (1924: 165); Leandri (1942: 280); Govaerts et al. (2000: 55).

\section{Acalypha claoxyloides Hutch., Bull. Misc. Inform. Kew 1918: 205.1918.}

Type. Seychelles, Astove, Cosmoledo and Aldabra, Apr 1907, H. P. Thomasset 243 (lectotype, designated here: K [K000186504]!).

Syntypes. Seychelles, Aldabra, s.d., W. L. Abbott s.n. P [P00887488]!, [P00887489]!; SeYchelles, Aldabra, Oct-Dec 1892, J. Fryer 18 K [K000186505]!.

Acalypha fryeri Hutch. Bull. Misc. Inform. Kew 1918: 206. 1918. Type. SEYCHELles, Aldabra, s.d., J. Fryer 92 (holotype: K [K000186506]!).

Acalypha aldabrica Pax \& K.Hoffm., Pflanzenr. 147,16(Heft 85): 136.1924.

Type. Seychelles, Aldabra, s.d., W. L. Abbott s.n. (holotype B?, presumably destroyed; lectotype, designated here: P [P00887488]!; isolectotype: P [P00887489]!, syn. nov.). 
Distribution. Seychelles Archipelago.

References. Hemsley (1919: 130); Hemsley (1919: 131) A. fryeri; Pax and Hoffmann (1924: 136) A. aldabrica; Fosberg (1974: 263); Renvoize (1975: 152); Robertson (1989: 199); Govaerts et al. (2000: 56).

Notes. Acalypha claoxyloides is widespread in the Seychelles archipelago. It is very close to A. pubiflora (Klotzsch) Baill., known from south-eastern Africa (Botswana, Malawi, Mozambique, South Africa and Zimbabwe), of which it may be a synonym. More studies of the African material, as well as the Australian material treated as $A$. pubiflora var. australica Radcl.-Sm. (Radcliffe-Smith 1990, 1996), is needed to unravel the A. pubiflora complex.

\section{Acalypha decaryana Leandri, Notul. Syst. (Paris) 10: 284. 1942.}

Type. Madagascar. Prov. Toliara: Ambovombe, 20 Aug 1924, R. Decary 2985 (holotype: P [P00508553]!; isotype W [W1962-0013399]!).

Distribution. MAdAgASCAR (Antananarivo, Antsiranana, Fianarantsoa, Toliara).

References. Govaerts et al. (2000: 59); Seebaluck et al. (2015: 150).

Notes. We consider as holotype of Acalypha decaryana, the only specimen with the word "type" hand-writen by Leandri.

\section{Acalypha diminuta Baill., Hist. Pl. Madag., Atlas, t. 194. 1891.}

Type. Plate 194 in Baillon ibid. loc., holotype.

Distribution. Madagascar (Antananarivo, Mahajanga, Toamasina, Toliara).

References. Baillon (1895b: 1197); Palacký (1907: 25); Pax and Hoffmann (1924: 21); Leandri (1935: 42); Leandri (1942: 253); Govaerts et al. (2000: 60).

Notes. Acalypha diminuta was first illustrated, without description, in Baillon's Histoire Naturelle des Plantes (Baillon 1891). The first description of this species, based only on Baillon's illustration, appears in Engler's Pflanzenreich (Pax and Hoffmann 1924). This is the only species included in Acalypha subgen. Androcephala Pax \& K.Hoffm.

\section{Acalypha emirnensis Baill., Adansonia 1: 270. 1861.}

Type. Madagascar. Prov. Antananarivo: Antananarivo "in prov. Emirna, prope Tananarivou", 1833, M. Bojer s.n. (holotype: P [P00536723]!; isotypes GD-C [GDC005713]!, P [P00536725]!).

Acalypha baronii Baker, J. Linn. Soc., Bot. 20: 254. 1883. Type: Madagascar: "Central Madagascar", 1882, R. Baron 1725 (holotype: K [K000186523]!; isotype P [P00324467]!). 
Acalypha hologyna Baker, J. Linn. Soc., Bot. 21: 441. 1885. Type: Madagascar: s.l., s.d., R. Baron 2889 (holotype: K [K000186526]!; isotype P [P00536724]!). Acalypha bakeriana Baill., Bull. Mens. Soc. Linn. Paris 2. 1180. 1895. Type: MADAGASCAR: "Centr. Madag." R. Baron 4425 (holotype: P [P00324466]!).

Acalypha emirnensis var. bara Leandri, Notul. Syst. (Paris) 10: 282. 1942. Type: Madagascar. Prov. Toliara: Massif of l'Ivakoany, 1928, H. Humbert 6986 (lectotype, designated here: P [P00508509]!; isolectotype: P [P00508508]! syn. nov.).

Syntypes. Madagascar. Prov. Toliara: bassin supérieur du Mandrare: col et sommet de Marosohy, 1000-1400 m, 14-15 Nov 1928, H. Humbert 6623 P [P00536758]!; Madagascar. Prov. Toliara: Massif de l'Ivakoany, H. Humbert 12185 P [P00508506!, P00508507]!; MadagasCar. Prov. Toliara: entre l'Andohahela et l'Elakelaka, H. Humbert 13941 [P00324472].

Acalypha emirnensis var. jabohaziana Leandri, Notul. Syst. (Paris) 10: 283. 1942. Type: Madagascar. Prov. Mahajanga: "Boina, Jabohazo, près du mont Tsitondroina" Dec 1900, H. Perrier de la Bâthie 9793 (holotype: P [P00536722]!. syn. nov).

Distribution. Madagascar (Antananarivo, Antsiranana, Fianarantsoa, Mahajanga, Toamasina, Toliara).

References. Müller Argoviensis (1866: 804); Baillon (1892: 1003); Baillon (1895a: 1180) as A. baronii; Baillon (1895b: 1196) as A. hologyna; Palacký (1907: 24, 25); Pax and Hoffmann (1924: 94, 171); Leandri (1942: 281); Govaerts et al. (2000: 50, 61); Govaerts et al. (2000: 67) sub. A. hologyna.

\section{Acalypha fasciculata Müll.Arg., Linnaea 34: 31. 1865.}

Type. Madagascar: s.l., s.d., L. M. A. Du-Petit Thouars s.n. (lectotype, designated here: P [P00324476]!; isolectotypes: P [P00324495!, P00508505!]).

Acalypha lyallii Baker, J. Linn. Soc., Bot. 20: 255. 1883. Acalypha fasciculata var. lyallii (Baker) Leandri, Notul. Syst. (Paris) 10: 284. 1942. Type: MadagasCAR: "Central Madagascar", s.d., R. Lyall s.n. (holotype: K [K000186529]! syn. nov.).

Acalypha madreporica Baill., Hist. Pl. Madag., Atlas t. 186 (1891). Type: Plate 186 in Baillon ibid. loc., holotype.

Acalypha madagascariensis Pax \& K.Hoffm., Pflanzenr. 147, 16 (Heft 85): 162. 1924. Type: Madagascar. Prov. Toliara: Forêt d'Antsianaka, 19 Jan 1882, $H$. Humblot 447 [ “449'] (holotype B, presumably destroyed; lectotype, designated here: P [P00324501]!, syn. nov.).

Acalypha fasciculata var. humbertiana Leandri, Notul. Syst. (Paris) 10: 284. 1942. Type: Madagascar. Prov. Toliara: haute vallée de Mandrare, 8 Nov 1928, $H$. Humbert 6514. Lectotype, designated here: P [P00508503]!; isolectotypes: P [P00324487]!, US [US00096332]! syn. nov.). Syntypes. MadagasCar. Prov. 
Toliara: Bassin de la Manampanihy (Sud-Est), col de Fitana, H. Humbert 6044 P [P00324486]!]; Madagascar. Prov. Toliara: Massif du Beampingaratra, du col de Bevava au sommet de Bekoho, H. Humbert 6478 P [P00508504]!.

Distribution. Madagascar (Antsiranana, Fianarantsoa, Mahajanga, Toamasina, Toliara).

References. Müller Argoviensis (1866: 851); Baillon (1895a: 1181) A. madreporica. and A. lyallii; Palacký (1907: 25); Pax and Hoffmann (1924: 94) A. madreporica; Pax and Hoffmann (1924: 171); Leandri (1942: 283); Leandri (1948: 186) A. lyallii; Leandri (1952) A. fasciculata var. humbertiana; Govaerts et al. (2000: 61); Govaerts et al. (2000: 73) A. lyallii; Govaerts et al. (2000: 74) A. madreporica; Schatz (2001: 142) as A. fasciculata var. humbertiana; Seebaluck et al. (2015: 152) as A. lyallii.

Notes. The correct number of the type specimen of $A$. madagascariensis is Humblot 447. In the protologue of this name, it is wrongly transcribed as "Humblot 449, which corresponds to a specimen of Psorospermum (Clusiaceae).

\section{Acalypha filiformis Poir., Encycl. 6(1): 205. 1804.}

Type. Mauritius, "Île de France", s.d., P. Commerson s.n. (lectotype, designated here: P [P05604464]!; isolectotypes: MPU [MPU014933]!, P [P05604471]!).

Acalypha filiformis var. arborea Poir., Encycl. 6(1): 205. 1804. Acalypha reticulata var. arborea (Poir.) Müll.Arg. Linnaea 34: 32. 1865. Type: Reunion, "Elle croît a l'île Bourbon", 1774, P. Commerson s.n. (holotype: P [P05604473]!; isotype: MPU [MPU014949]!).

Tragia reticulata Poir., Encycl. 7: 725. 1806. Acalypha reticulata (Poir.) Müll.Arg., Prodr. 15(2): 851. 1866. Type: Reunion: "I' Ile -de-Bourbon", s.d., P. Commerson s.n. (holotype: P-LAM [P00382118]!; isotype P [P05604477]!).

Acalypha arborea Commers. in Poir., Encycl. 6: 205.1804 nom. nud.

Acalypha lantanaefolia Bojer, Hortus Maurit. 286. 1837 nom. nud.

Distribution. Mascarene Islands (Mauritius, Réunion).

References. Bojer (1837: 286); Baillon (1858: 443) as Tragia reticulata; Baillon (1861: 266) as A. arborea; Müller Argoviensis (1866: 851) as A. reticulata; Baker (1877: 316) as $A$. reticulata; Müller Argoviensis (1882: 26) as $A$. reticulata; Pax (1890: 61) as A. reticulata; $\operatorname{Pax}(1894: 96)$ as A. reticulata; de Cordemoy (1895: 342) as $A$. reticulata; Voeltzkow (1917: 447) as $A$. arborea; Pax and Hoffmann (1924: $102)$ as $A$. reticulata; Leandri (1942: 258) as A. reticulata; Coode (1979: 45) as A. reticulata; Coode (1982: 69, 76); Govaerts et al. (2000: 62, 99, 105); Seebaluck et al. (2015: 150).

Notes. Acalypha reticulata has been usually considered as the accepted name of this species. Leandri (1942) noticed that $A$. filiformis and $A$. reticulata are conspecific, but he kept $A$. reticulata as the accepted name. Applying the rule of priority, the accepted name must be $A$. filiformis and $A$. reticulata should be placed as a synonym. 


\section{Acalypha fimbriata Schumach. \& Thonn., Beskr. Guin. Pl. 409. 1827.}

Type. Ghana: s.l., s.d., P. Thonning s.n. (holotype: C [C10003279]!; isotypes: C [C10003278!, C10003280]!, S [S14-42539]!).

Distribution. West Tropical Africa, West Central Tropical Africa, East Tropical Africa, Northeast Tropical Africa and Southern Africa. Madagascar (Antananarivo, Fianarantsoa, Toliara).

Notes. This is the first time that this species is cited for the WIOR region, where it is almost certainly introduced.

\section{Acalypha gracilipes Baill., Adansonia 1: 273. 1861.}

Acalypha commersoniana var. gracilipes (Baill.) Müll.Arg., Prodr. 15(2): 850. 1866. Acalypha integrifolia var. gracilipes (Baill.) Pax \& K.Hoffm., Pflanzenr. 147,16 (Heft 85): 106. 1924.

Type. Madagascar: s.l., s.d., P. Commerson s.n. (holotype: P [P04022747]!). Acalypha reticulata var. cloiselana Leandri, Notul. Syst. (Paris) 10: 266.1942. Type: Madagascar: s.l., s.d., P. Commerson s.n. (lectotype, designated here: P [P00513166]!, syn. nov.).

Syntypes. Madagascar. Prov. Toamasina: Fénérive, H. Perrier de la Bâthie 9707 [P00513169]!; Madagascar. Prov. Toliara: Fort Dauphin, J. Cloisel 156 P [P00513165]!; Madagascar. Prov. Toliara: Forêt de Manantantely, $H$. Humbert 5835 P [P00513167]!, P [P00513168]!; Madagascar. Prov. Toliara: Fort Dauphin, G. F. Scott-Elliot 2493 P [P00513171]!; MadaGASCAR: s.l., s.d., R. Baron 5980 P [P00513164]!; R. Baron 6420 [P00324562]!.

Acalypha cloiselana Denis ex Leandri, Notul. Syst. (Paris) 10: 266. 1942 nom. nud. as synonym of $A$. reticulata var. cloiselana Leandri.

Acalypha commersonii Baill. ex Leandri, Notul. Syst. (Paris) 10: 266. 1942 nom. nud. as synonym of $A$. reticulata var. cloiselana Leandri.

Distribution. MADAGASCAR (Antsiranana, Fianarantsoa, Toamasina, Toliara).

References. Müller Argoviensis (1866: 850) as A. commersoniana var. gracilipes; Baillon (1892: 1004); Palacký (1907: 25); Pax and Hoffmann (1924: 106) as A. integrifolia var. gracilipes; Govaerts et al. (2000: 69, 100).

Notes. Acalypha gracilipes has been usually treated as a variety of $A$. integrifolia Willd., which is endemic of Mascarene Islands, but $A$. gracilipes can be clearly distinguished by its elliptic-lanceolate leaves, denticulate female bracts and glabrous and glandular ovaries, vs. linear-lanceolate leaves, entire female bracts and hispidulous and echinate ovary in A. integrifolia.

A. gracilipes can be distinguished from both $A$. urophylla Boivin ex Baill. and $A$. paxii Aug.D.C. mainly by its glabrous leaves with crenate to subdentate margins and obtuse to subacute apices, glabrous female bracts and glabrous ovaries with minute sessile glands, vs. pubescent leaves with serrate margins and acuminate (A. urophylla) or 
usually caudate $(A$. paxii) apices, pubescent female bracts and hispidulous ovaries with long papillae. In addition, the female bracts of $A$. paxii have a prominent central tooth, which is absent in both $A$. gracilipes and $A$. urophylla.

Leandri (1942) included the collection H. Perrier de la Bâthie 9746 as Acalypha reticulata var. cloiselana Leandri, however the specimen of this collection in $\mathrm{P}$ (P00513170) corresponds to A. urophylla.

\section{Acalypha hispida Burm.f., Fl. Ind. 303, pl. 61, f. 1. 1768.}

Ricinocarpus hispidus (Burm. f.) Kuntze, Revis. Gen. Pl. 2: 618. 1891.

Type. Habitat in India, Tab. 61 in Burm. f., loc. cit. 302. 1768.

Distribution. Introduced in Tropical Africa and the WIOR. MadagasCar (Toliara).

References. Bojer (1837: 25); Baillon (1861: 274); Palacký (1907: 25); Robertson (1989: 199).

Notes. This shrub, native to Melanesia or Malesia, is frequent in gardens throughout the tropics and rarely appears naturalised. As all plants are pistillate, it can only reproduce clonally. We found collections from Madagascar and Seychelles where it is cultivated. It has been reported from Madagascar (Palacký 1907), Mauritius (Bojer 1837; Baillon 1861), Réunion (Baillon 1861: 274) and the Seychelles (Robertson 1989).

\section{Acalypha humbertii Leandri, Notul. Syst. (Paris) 10: 274.1942.}

Type. Madagascar. Prov. Toliara: vallées du Mangoky et de l'Isahaina, aux environs de Beroroha, 200 m, Oct 1933, H. Humbert 11289 (lectotype, designated here: P [P00508400]!; isolectotype: P [P00508399]!).

Syntypes. Madagascar. Prov. Toliara: Bassin supérieur de Mandrare du Sud-Est, entre le col de Vavara et la vallée de la Manambolo, 700-1200 m, 20-22 Dec. 1928, H. Humbert 6758 P [P00508401!, P00508402!, P00508403!, P00508404]!).

Distribution. Madagascar (Toliara).

References. Govaerts et al. (2000: 67).

\section{Acalypha indica L., Sp. P1. 2: 1003. 1753.}

Type. India: s.l., s.d., Herb. Hermann 3: 2, \#34. (lectotype, designated by RadcliffeSmith (1986: 65): BM; isolectotype: BM).

Distribution. Widely distributed in the Paleotropics and introduced in the Americas. In the WIOR, it is found only in disturbed areas and almost certainly is introduced there. Madagascar (Antananarivo, Antsiranana, Fianarantsoa). Comoros Archipelago (Anjouan, Mohéli, Mayotte). Mascarene Islands (Mauritius, Réunion). Seychelles Archipelago. 
References. Bojer (1837: 285); Baillon (1861: 274); Müller Argoviensis (1865: 42; 1866: 868); Baker (1877: 314); Müller Argoviensis (1882: 27); Baillon (1895b: 1197); de Cordemoy (1895: 342); Palacký (1907: 25); Voeltzkow (1917: 447); Hemsley (1919: 148); Pax and Hoffmann (1924: 33); Leandri (1935: 43); Leandri (1942: 256); Renvoize (1975: 152); Coode (1982: 69, 78); Robertson (1989: 200); Govaerts et al. (2000: 68).

\section{Acalypha integrifolia Willd., Sp. Pl. 4(1): 530. 1805.}

Type. Mauritius, s.l., s.d., Anonymous, s.n. (lectotype designated by Coode (1978: 39): B [B-W17834-020]).

Tragia colorata Poir., Encycl. 7: 725. 1806. Acalypha colorata (Poir.) Spreng., Syst. Veg. 3: 879. 1826. Acalypha commersoniana var. obtusifolia f. colorata (Poir). Müll.Arg., Prodr. 15(2): 850. 1866. Acalypha integrifolia var. colorata (Poir.) Pax \& K.Hoffm. Pflanzenr. 147,16 (Heft 85): 106. 1924. Type: Mauritius: "Cete plante croit dans les indes orientales, \& à l'Ile de France,", s.l. s.d., P. Commerson s.n. (holotype: P-LAM [P00382140]!).

Acalypha reticulata var. longifolia Müll.Arg., Linnaea 34: 32. 1865. Acalypha integrifolia var. longifolia (Müll.Arg.) Coode, Kew Bull. 34: 41. 1979. Type: MaURITIus: s.l., s.d., L. Bouton s.n. (holotype: GDC [G00324522]!, syn. nov.).

Acalypha reticulata var. longifolia f. aberrans Müll.Arg., Linnaea 34: 32. 1865. Type: Mauritius: "In sylvis Mauritii", 1833, M. Bojer s.n. (holotype: GDC [G00324521]!).

Acalypha commersoniana Baill. ex Müll.Arg., Prodr. 15(2): 849. 1866. Acalypha commersoniana var. brevifolia Baill. ex Müll.Arg., Prodr. 15(2): 850. 1866. Type: Mauritius: "Cum var. praecedentibus", s.d., Anonymous, s.n. (Hb. Willd. fol. 17834 pag. 1) (lectotype, designated here: B [B-W17834-010]!).

Syntypes. Mauritius: s.l., s.d., L. Bouton s.n. GDC [G00324543]!.

Acalypha commersoniana var. acutifolia Müll.Arg., Prodr. 15(2): 849. 1866. Acalypha commersoniana var. acutifolia f. purpurea Müll.Arg., Prodr. 15(2): 849. 1866. Type: Mauritius: s.l., s.d., F.W. Sieber 181 pr. p. (lectotype, designated here: GDC [G00324550]!). Syntype: Mauritius: s.l., s.d., L. Bouton s.n. GDC [G00324559]!.

Acalypha commersoniana var. acutifolia f. purpureo-marginata Müll.Arg., Prodr. 15(2): 849. 1866. Type: Mauritius: s.l., s.d., Hb. Boiss. s.n. (holotype: GDC [G00324557]!; isolectotype: K [K000431097]!, syn. nov.).

Acalypha commersoniana var. acutifolia f. concolor Müll.Arg., Prodr. 15(2): 849. 1866. Acalypha integrifolia var. concolor (Müll.Arg.) Pax \& K.Hoffm, Pflanzenr. 147,16 (Heft 85): 106. 1924. Type: Mauritius: "Cum form. Praecentibus", s.d., J. B. G. M. Bory s.n. (lectotype, designated here: GDC [G00324554]!). Syntype: Mauritius: s.l., s.d., F. W. Sieber 182 GDC [G00324556]!. 
Acalypha commersoniana var. longifolia Müll.Arg., Prodr. 15(2): 850. 1866. Type: Mauritius: s.l., s.d., Bouton s.n. (lectotype, designated here: GDC [G00324553]!; isolectotypes: GDC [G00324552!, G00324551!], K [K000431101!], syn. nov.).

Acalypha commersoniana var. parvifolia Baill. ex Müll.Arg., Prodr. 15(2): 850. 1866. Acalypha integrifolia var. parvifolia (Baill. ex Müll.Arg.) Pax \& K.Hoffm., Pflanzenr. 147,16(Heft 85): 106. 1924. Type: Mauritius: "Cum var. praecedentibus", s.d., F. W. Sieber 369 p.p. (holotype: GDC [G00324538]!, syn. nov.).

Acalypha commersoniana var. obtusifolia Müll.Arg., Prodr. 15(2): 850. 1866. Acalypha commersoniana var. obtusifolia f. discolor Müll.Arg., Prodr. 15(2): 850. 1866. Type: Mauritius: "Cum praecedentibus", s.d., F. W. Sieber 181 pr. $p$. (holotype: GDC [G00324558]!, syn. nov.).

Acalypha commersoniana var. obtusifolia f. unicolor Müll.Arg., Prodr. 15(2): 850. 1866. Type: Mauritius: s.l., s.d., F. W. Sieber 178 (holotype: GDC [G00324539]!; isotypes P [P04779345!, P04780015!].

Acalypha discolor Bojer, Hortus Maurit. 286. 1837 nom. nud. Tragia macrophylla Wall., Numer. List n. 7796. 1847 nom. nud.

Tragia lobata Wall., Numer. List n. 7796. 1847 nom. nud. Acalypha commersoniana Baill., Adansonia 1: 267. 1861 nom. nud. Acalypha commersoniana var. concolor Baill., Adansonia 1:267. 1861 nom. nud. Acalypha commersoniana var. discolor Baill., Adansonia 1: 267. 186 nom. nud. Acalypha commersoniana var. parvifolia Baill., Adansonia 1:267. 1861 nom. nud. Caturus sessilis Pet. Thou. ex Baill., Adansonia 1:267. 1861 nom. nud. Tragia fruticosa Commers. ex Baill., Adansonia 1:267. 1861 nom. nud. Tragia obtusata Vahl. ex Baill., Adansonia 1: 267. 1861 nom. nud.

Distribution. Mascarene Islands (Mauritius, Réunion).

References. Bojer (1837: 286); Baillon (1858: 443) A. colorata; Müller Argoviensis (1866: 850); Baker (1877: 315) A. colorata; de Cordemoy (1895: 342) A. colorata; Palacký (1907: 24) A. commersoniana; Palacký (1907: 25); Pax and Hoffmann (1924: 105); Coode (1982: 69); Robertson (1989: 200); Govaerts et al. (2000: 69).

Notes. Coode (1982) accepted three subspecies with six varieties within Acalypha integrifolia, but we find the varieties he placed within subsp. integrifolia to overlap too much to accept as distinct taxa. See $A$. marginata (Poir.) Spreng. for our treatment of what Coode treated within A. integrifolia subsp. marginata (Poir.) Coode and subsp. panduriformis Coode.

\section{Acalypha lamiana (Leandri) I.Montero \& Cardiel., comb. nov. urn:Isid:ipni.org:names:60476986-2}

Acalypha reticulata var. urophylla f. lamiana Leandri, Notul. Syst. (Paris) 10: 263.1942. 
Type. Madagascar, Prov. Mahajanga, Reserve de Marohogo, 28 Dec 1938, H. J. Lam \& A. D. J. Meeuse, 6127 (lectotype, designated here: P [P05604417]!; isolectotype: L [L0242109]!).

Syntypes. Madagascar, Prov. Antananarivo, Tsarasaotra, Feb 1898, H. Perrier de la Bâthie 457 P [P05604408!, P05604409!, P05604410!, P05604413!]; Madagascar, Prov. Fianarantsoa, Ankirihitra près du mont Tsilondroina, Mar 1902, H. Perrier de la Bâthie 9817 P [P05604403]!, 9817 bis P [P05604404!, P05604405!]; MadaGasCAR, Prov. Mahajanga, NW of Ankazobe,Vallée de l'Ikopa, 14 Mar 1930, R. Decary 7535 P [P05604421]!; Massif de l'Ankarafantsika, 11 Jan 1938, R. Decary 12876 P [P05604420]!; Bekodoka, 17 Sept 1930, R. Decary 8109 P [P05604422]!; Region d'Antsalova, 1932-1933, J. Leandri 998 P [P05604411]!; Tsingy du Bemaraha, 3-6 Oct 1932, J. Leandri 176 P [P05604415]!; Dokolahy, Feb-Apr 1933, J. Leandri 602 P [P05604414]!; MadagasCar, Prov. Toliara, Soahazo Forest, 100 m, 22 Oct 1932, J. Leandri 414 P [P05604416]!.

Distribution. Madagascar (Antananarivo, Fianarantsoa, Mahajanga and Toliara).

Notes. Acalypha lamiana was treated by Leandri (1942) as a form of $A$. reticulata var. urophylla (Boivin ex Baill.) Müll.Arg. (treated here as $A$. urophylla). After studying the numerous type collections, it seems clear to us that $A$. lamiana must be considered as a distinct species. A. lamiana differs from $A$. urophylla mainly by the leaves that are rounded at the base and reddish at the margins and its subentire, eglandular female bracts vs. leaves that are usually acute at the base and not reddish at the margins and dentate female bracts with small sessile glands at margins, in $A$. urophylla.

One of the mentioned syntypes, Perrier de la Bâthie 9817, was wrongly transcribed by Leandri (1942: 263) as " 8917 ".

\section{Acalypha lanceolata var. glandulosa (Müll.Arg.) Radcl.-Sm., Kew Bull. 44(3): 444. 1989.}

Acalypha crenata var. glandulosa Müll.Arg., Linnaea 34: 43. 1865.

Type. Tanzania: Zanzibar, 1847-1852, L. H. Boivin s.n. (lectotype, designated here: $\mathrm{P}$ [P05511211]!; isolectotypes: P [P05511212!, P05511225!, P05510174!]).

Distribution. Acalypha lanceolata Willd. is widely distributed in the Paleotropics; the var. glandulosa occurs in East Tropical Africa and South Tropical Africa. MadAGASCAR (Antsiranana). Mascarene IsLands (Réunion).

References. Müller Argoviensis (1866: 872); Coode (1982: 69, 79); Govaerts et al. (2000: 71); Seebaluck et al. (2015: 152).

Notes. Acalypha lanceolata var. lanceolata occurs in Asia. The main diference between the African var. glandulosa and the Asian var. lanceolata is the presence or absence of stipitate glands. Additional studies are needed to clarify the taxonomic status of these taxa. 
19. Acalypha leonii Baill., Bull. Mens. Soc. Linn. Paris ii. (1895) 1197.

Type. Madagascar. Prov. Toamasina: Forêt d'Antsianaka, 14 Dec 1882, L. Humblot 514 (lectotype, designated here: P [P00513056]!; isolectotypes: K [K000186528]!, P [P00513057!, P00513058!, P00513055!]).

Acalypha leonii var. perrierana Leandri, Notul. Syst. (Paris) 10: 271. 1942. Type: Madagascar. Prov. Mahajanga: bassin du Bemarivo, versant NE, $100 \mathrm{~m}$, 1912, H. Perrier de la Bâthie 9719 (lectotype, designated here: P [P00513061]!; isolectotypes: P [P00513059!, P00513060!], syn. nov.).

Distribution. Madagascar (Antananarivo, Antsiranana, Toamasina, Toliara).

References. Palacký (1907: 25); Pax and Hoffmann (1924: 112); Leandri (1942: 271); Leandri (1952); Govaerts et al. (2000: 72); Schatz (2001: 142).

20. Acalypha lepidopagensis Leandri, Notul. Syst. (Paris) 10: 280.1942.

Type. Madagascar. Prov. Antsiranana: Massif du Tsaratanana, 1000 m, Dec 1912, H. Perrier de la Bâthie 9726 (lectotype, designated here: P [P00513062]!; isolectotype: P [P00513063]!).

Distribution. Madagascar (Antsiranana).

References. Leandri (1952); Govaerts et al. (2000: 72); Schatz (2001: 142).

\section{Acalypha leptomyura Baill., Hist. P1. Madag., Atlas (1891) t. 191.}

Type. Madagascar. Plate 191 in Baillon ibid. loc., holotype.

Distribution. Madagascar (Antananarivo, Antsiranana, Fianarantsoa, Mahajanga, Toamasina and Toliara).

References. Baillon (1892: 1004); Koehne (1892: 131); Palacký (1907: 25); Pax and Hoffmann (1924: 112); Leandri (1942: 271); Govaerts et al. (2000: 72).

\section{Acalypha linearifolia Leandri, Notul. Syst. (Paris) 10: 275.1942.}

Type. Madagascar. Prov. Toliara: Ambovombe, Kotoala, 21 Jan 1931, R. Decary 8423 (lectotype, designated here: P [P00513090]!; isolectotypes: S [S07-14664]!, TAN [TAN000510]!, US [US01014148]!).

Syntypes. Madagascar. Prov. Toliara: delta de la Linta, 17-24 Aug 1928, H. Humbert \& C. F. Swingle 5385 P [P00513086!, P00513087!, P00513088!, P00513089!]; US [US00096361]!.

Distribution. MADAgascar (Toamasina, Toliara).

References. Govaerts et al. (2000: 72). 


\section{Acalypha marginata (Poir.) Spreng., Syst. Veg. 3: 879. 1826.}

Tragia marginata Poir., Encycl. 7: 725. 1806. Acalypha integrifolia subsp. marginata

(Poir.) Coode, Kew Bull. 34: 42.1979.

Type. Mauritius: "Les Indes Orientales", s.d. (holotype P-LAM [P00382145]!).

Acalypha integrifolia subsp. panduriformis Coode, Kew Bull. 34: 42. 1979. Type: Réunion: Cliff between St Philippe and St Joseph near Basse vallée, ca. $100 \mathrm{~m}$, 26 Feb 1975. M. J. E. Coode \& T. H. Cadet 4968 (holotype K [K000431108]!; isotype K [K000431107]!, syn. nov.).

Acalypha integrifolia subsp. marginata var. saltuum Coode, Kew Bull. 34: 43. 1979. Type: Mauritius: Macabé, 650 m, 15 Feb 1975, M. J. E. Coode et al. 4874 (holotype: K [K000431106]!; isotypes P [P04779351]!, MAU [n.v.], syn. nov.).

Acalypha integrifolia subsp. marginata var. crateriana Coode, Kew Bull. 34: 44. 1979. Type: Mauritius: Tamarin Falls, 2 Mar1975, D. Lorence 1138 (holotype: K [K000431104]!; isotype K [K000431105]!, MAU [n.v.], syn. nov.).

Distribution. Mascarene Islands (Mauritius, Réunion).

References. Bojer (1837: 286); Baillon (1858: 443); Baillon (1861: 267); Baker (1877:315); de Cordemoy (1895: 343); Palacký (1907: 25); Pax and Hoffmann (1924: 106); Coode (1982: 73) A. integrifolia subsp. panduriformis, A. integrifolia subsp. marginata; Coode (1982: 74) A. integrifolia var. saltuum, A. integrifolia var. crateriana; Govaerts et al. (2000:69) A. integrifolia subsp. marginata; Seebaluck et al. (2015: 152).

Notes. Acalypha marginata was treated by Coode (1979) as A. integrifolia subsp. marginata. We consider $A$. marginata to be a distinct species, differentiated mainly by its variegated leaf blades and vestigial female bracts that are not accrescent in fruit vs. non variegated leaf blades and conspicuous female bracts that are accrescent in fruit in A. integrifolia. We include as synonyms $A$. integrifolia subsp. panduriformis, $A$. integrifolia var. crateriana and $A$. integrifolia var. saltuum because they have the same characters as $A$. marginata.

24. Acalypha medibracteata Radcl.-Sm. \& Govaerts, Kew Bull. 52(2): 477. 1997. Acalypha gagnepainii Leandri Notul. Syst. (Paris) 10: 274.1942 nom. illeg. non $A$. gagnepainii Merr. (1938).

Type. Madagascar. Prov. Toliara: Massif du Vohitsiombe (Fort-Dauphin), 31 Jul 1926, R. Decary 4664 (lectotype, designated here: P [P00508417]!; isolectotype: S [S07-14667]!).

Syntypes. Madagascar. Prov. Toliara: Vallée du Mandrare, s.d., R. Decary 2620 P [P00508418]!; Madagascar. Prov. Toliara: Vallée de l'Ikonda, au N. d'Ambovombe, R. Decary 8913, P [P00887487]!; Madagascar. Prov. Toliara: Imangory, s.d., R. Decary 8948 P [P00508416]!; MadagasCar. Prov. Toliara: Beteny, (limite Nord-Est de 
l'Androy), 22 Nov 1931, R. Decary 9355 P [P00508415]!, G [G00034242]!, GB [GB0047682]!.

Acalypha medibracteata var. calcicola (Leandri) Radcl.-Sm. \& Govaerts, Kew Bull. 52(2): 477. 1997. Acalypha gagnepainii var. calcicola Leandri Notul. Syst. (Paris) 10: 275. 1942. Type: Madagascar. Prov. Toliara: vallée moyenne du Mandrare près d'Anadabolava, Dec 1933, H. Humbert 12422 (lectotype, designated here: P [P00508409]!; isolectotypes: P [P00508405!, P00508406!, P00508407!, P00508408!], syn. nov.).

Syntypes. Madagascar. Prov. Toliara: Fort-Dauphin, J. Cloisel $18 \mathrm{P}$ [P00508410]!, P [P00508411]!; Madagascar. Prov. Toliara: Basse vallée du Fiherenana, s.d., H. Humbert 11573 P [P00508412!, P00508413!].

Acalypha menabeana Leandri Notul. Syst. (Paris) 10: 275.1942 nom. nud. as $A$. gagnepainii.

Acalypha aspretorum Leandri Notul. Syst. (Paris) 10: 275. 1942 nom. nud. as $A$. gagnepainii.

Distribution. Madagascar (Antananarivo, Toamasina, Toliara).

References. Govaerts et al. (2000: 75, 100).

\section{Acalypha menavody (Leandri) I.Montero \& Cardiel, comb. nov.} urn:Isid:ipni.org:names:60476988-2

Acalypha spiciflora var. menavody Leandri, Notul. Syst. (Paris) 10:270. 1942.

Type. Madagascar. Prov. Antsiranana: Collines et plateaux calcaires de l'Analamera, Jan 1938, H. Humbert 19149 (lectotype, designated here: P [P00536737]!; isolectotypes: P [P00536736!, P00536738!]).

Distribution. Madagascar (Antsiranana).

Notes. Acalypha spiciflora has been excluded because it does not belong to Acalypha (see notes under this name). We only recognise A. spiciflora var. menavody as A. menavody.

\section{Acalypha paxii Aug.D.C., Bull. Herb. Boissier, sér. 2, 1: 567. 1901.}

Acalypha urophylla Pax, Bot. Jahrb. Syst. 19: 96. 1894. nom. illeg. non A. urophylla Boivin ex Baill. (1861). Acalypha reticulata var. urophylloides Pax \& K.Hoffm., Pflanzenr. 147, 16 (Heft 85): 105. 1924. Acalypha filiformis var. urophylloides (Pax \& K.Hoffm.) Govaerts, World Checkl. Bibliogr. Euphorbiaceae 63. 2000.

Type. Madagascar. Prov. Antsiranana: Nossibé, nordwest, Feb 1880, J. M. Hildebrandt 3356 (holotype B or WRSL, presumably destroyed; lectotype, designated here: BREM [BREM0001784]!; isolectotypes: JE [JE00004294!, JE00004293!], K [K000186531]!, M [M0110600]!, P [P00536741!, P00536742!, P00536743!]). 
Acalypha pervilleana Baill., Adansonia 1:273. 1861. Acalypha reticulata var. pervilleana (Baill.) Müll.Arg., Linnaea 34: 32. 1865. Acalypha filiformis var. pervilleana (Baill.) Govaerts, World Checkl. Bibliogr. Euphorbiaceae 62. 2000. Type. Madagascar. Prov. Antsiranana: Nossibé, 1840, M. Richard 384 (lectotype, designated here: P [P00536745]!; isolectotype: P [P05604474]!, syn. nov.).

Syntypes. Madagascar, Prov. Antsiranana: Nossibé, M. Pervillé 368 P [P00536746!, P00536747!].

Acalypha humblotiana Baill., Hist. Pl. Madag., Atlas (1891) t. 1891. Acalypha reticulata var. urophylla f. humblotiana (Baill.) Leandri, Notul. Syst. (Paris) 10: 262. 1942. Type: Comoros: Grande comore, 14 Nov 1885, L. Humblot 1461 (lectotype, designated here: P [P00196295]!; isolectotypes: P [P02712292!, P00196296!], syn. nov.).

Acalypha meiodonta Baill., Bull. Mens. Soc. Linn. Paris 2: 1197. 1895. Acalypha reticulata var. meiodonta (Baill.) Leandri. Notul. Syst. (Paris) 10: 267. 1942. Type: Madagascar: Centr. Madag., Dec. 1883, R. Baron 2826 (lectotype, designated here: P [P05604378]!; isolectotype: K [K000186508]!, syn. nov.). Syntype: Madagascar: “Centr. Madag.", s.d., R. Baron 6581 K [n.v.].

Acalypha reticulata var. meiodonta f. andronea Leandri, Notul. Syst. (Paris) 10: 268. 1942. Type: Madagascar, Prov. Mahajanga, Bemarivo (Boïna), Dec 1906, $H$. Perrier de la Bâthie 9561 (lectotype, designated here: P [P00513143]!; isolectotype: P [P00513144]!, syn. nov.).

Former syntypes: Madagascar, Prov. Mahajanga, bord du massif du Manongarivo, versant du Sambirano, 1909, H. Perrier de la Bâthie 9934 P [P00513149]!; Massif du Manongarivo, versant du Sambirano, Sep 1909, H. Perrier de la Bâthie 9939 P [P00513150]!; haut Bemarivo (Andranofosy), Boïna, Jan 1907, H. Perrier de la Bâthie 9635 P [P00513145]!; Massif du Manongarivo, Sambirano, Apr 1909, H. Perrier de la Bâthie 9928 P [P00513142]!; Manongarivo (Ambongo), Oct 1904, H. Perrier de la Bâthie 1677, P [P00513146!, P00513147!].

Distribution. Madagascar (Antsiranana, Mahajanga, Toamasina). Comoros Archipelago (Grande Comore, Anjouan, Mohéli and Mayotte).

References. Müller Argoviensis (1866: 852) as A. reticulata var. pervilleana; Müller Argoviensis (1882: 26) as A. reticulata var. urophylla; Müller Argoviensis (1866: 852) as $A$. reticulata var. pervilleana; Baillon (1892: 1004) as $A$. pervilleana; Baillon (1895b: 1197) as A. humblotiana; Palacký (1907: 25, 26); Pax and Hoffmann (1924: 105, 112) as Acalypha reticulata var. urophylloides and $A$. reticulata var. pervilleana; Leandri $(1942: 258,260)$ as A. reticulata var. pervilleana; Govaerts et al. (2000: 62, 63, 75, 104 105, 107).

Notes. Acalypha paxii was proposed by August De Candolle as a replacement name for the illegitimate $A$. urophylla Pax. Leandri (1942) treated $A$. paxii as a synonym of A. reticulata var. pervilleana. We consider that $A$. paxii is a well-differentiated species.

Acalypha paxii, A. pervilleana and $A$. meiodonta have been considered synonyms of $A$. reticulata, which we consider to be a synonym of $A$. filiformis. $A$. paxii can be differentiated from $A$. filiformis by its sessile, dentate female bracts with a prominent 
central tooth vs. pedicellate, crenate to subentire female bracts in $A$. filiformis (see notes in A. filiformis).

Acalypha paxii can be distinguished from $A$. urophylla, which has also been placed within $A$. reticulata, mainly by its leaves with usually caudate apices, its dentate female bracts with a prominent central tooth and eglandular margins vs. leaves with usually acuminate apices, dentate female bracts without a prominent central tooth and with small sessile glands at the margins. See notes under $A$. gracilipes Baill. for the differences between $A$. paxii and that species.

Acalypha humblotiana was first illustrated, without description, in Baillon`s (1891) Histoire Naturelle des Plantes, but this illustration is not consistent with Baillon's (1895b) later description nor with the specimen on which the description presumably is based (L. Humblot 1461).

Although Richard 384 [P00536745]! and 385 [P04779454]! have labels giving the locality as "Bourbon", now Réunion, these are not Richard's original labels and the species is otherwise unknown from the Mascarene Islands. We do not have evidence that it occurs there.

\section{Acalypha perrieri Leandri, Notul. Syst. (Paris) 10: 273.1942.}

Type. Madagascar. Prov. Mahajanga: Belambo, près de Maevatanana, Aug 1901, H. Perrier de la Bâthie 981 (lectotype, designated here: P [P00513095]!).

Syntypes. Madagascar. Prov. Mahajanga: Menabé, Tsiampihy, J. Leandri 275 P [P00513092]!, P [P00513093]!, TAN [TAN000511]!; Madagascar. Prov. Mahajanga: ibid. loc., s.d., J. Leandri 294, P [P00513091]!; Madagascar. Prov. Mahajanga: Forêt de Tsimembo, s.d., J. Leandri 420, P [P00513094]!.

Distribution. Madagascar (Antsiranana, Mahajanga, Toliara).

References. Govaerts et al. (2000: 81).

\section{Acalypha poiretii Spreng., Syst. Veg. 3: 879. 1826.}

Ricinocarpus poiretii (Spreng.) Kuntze, Gen. Pl. 2: 618. 1891.

Type. “Amer. trop.” s. loc., s.d., Anonymous s.n. (holotype: P-LAM [P00382110]!).

Distribution. Introduced in Tropical Africa and the WIOR region. Mascarene Islands (Mauritius, Réunion, Rodríguez).

References. Baker (1877: 315); de Cordemoy (1895: 312); Coode (1982: 79).

Notes. Herb native to the Americas. It has been reported from continental Africa (Cardiel and Montero Muñoz 2018) and from the Mascarene Islands (Baker 1877; de Cordemoy 1895; Coode 1982). We found specimens from the Mascarene Islands (Mauritius, Réunion and Rodríguez). 


\section{Acalypha radula Baker, J. Linn. Soc., Bot. 20: 254.1883.}

Type. Madagascar: "Central Madagascar", 1882, R. Baron 1818 (holotype: K [K000186509]!; isotypes: P [P00513119!, P00513120!]).

Acalypha hildebrandtii Baill., Bull. Mens. Soc. Linn. Paris 2: 1005, 1180. 1892. Type: Madagascar. Prov. Fianarantsoa: "Betsileo, Nandahizana,". J. M. Hildebrandt 3900 (holotype: P [P00513121]; isotypes: BREM [BREM0001783]!, G [G00190630!, G00074184!], JE [JE00000289!, JE00000288!], K [K000186507]!, M [M0110604]!, P [P00513122]!).

Acalypha andringitrensis Leandri, Notul. Syst. (Paris) 10: 277. 1942. Type. Madagascar. Prov. Fianarantsoa: Massif of Andringitra, Apr 1921, H. Perrier de la Bâthie 13640 (lectotype, designated here: P [P00508596]!; isolectotypes: P [P00508594!, P00508595]!, syn. nov.).

Syntypes. Madagascar, ibid. loc., 1924, H. Humbert 3709 P [P00224706]!, P [P00508591]!, P [P00508592]!, P [P00508593]!; Madagascar, ibid. loc., Apr 1921, H. Perrier de la Bâthie 9671 P [P00508589!, P00508590!].

Acalypha salviifolia Baill., Étude Euphorb. 443. 1858 nom. nud.

Tragia salviaefolia Boj. ex Baill., Étude Euphorb. 443. 1858 nom. nud.

Distribution. Madagascar (Antananarivo, Fianarantsoa, Toamasina and Toliara).

References. Baillon (1861: 268) as A. salviifolia; Müller Argoviensis (1866: 889) A. salviifolia; Baillon (1891: 193); Baillon (1892: 1004) A. salviifolia; Baillon (1895a: 1180) A. hildebrandtii; Palacký (1907: 25) A. hildebrandtii; Palacký (1907: 26); Palacký (1907: 26) A. salviifolia; Pax and Hoffmann (1924: 102) as A. salviifolia; Pax and Hoffmann (1924: 156) A. hildebrandtii; Leandri (1942: 278); Leandri (1952); Jenkins (1987:347); Jenkins (1990: 408, 433); Goodman (1996: 61) A. andringitrensis; Govaerts et al. (2000: 49) A. andringitrensis; Govaerts et al. (2000: 67) A. hildebrandtii; Govaerts et al. (2000: 85, 86, 105) A. salviifolia; Schatz (2001: 142); Seebaluck et al. (2015: 149, 153).

Notes. Although Leandri (1942) distinguished Acalypha andringitrensis and A. radula based on leaf shape and bract incision, more recent collections show continuous variation between the extremes recognised by Leandri, and DNA sequences do not differentiate these forms (G. A. Levin, pers. obs.).

\section{Acalypha richardiana Baill., Adansonia 1: 268. 1861.}

Type. Comoros: Mohéli, "Ile Mohilla”, s.d., M. Richard 287 (lectotype, designated here: P [P04779566]!; isolectotypes: P [P04779562!, P04779564!, P04779565!]).

Syntypes. Madagascar: s.l., s.d., M. Richard 544 [P04779563]!; Mayotte, s.l., s.d., L. H. Boivin 3373 GDC [G00324505]!, P [P00196299!, P00196300!], W [WRchb. 1889-0166704]!.

Acalypha ovalifolia Baill., Adansonia 1: 269. 1861. Acalypha reticulata var. ovalifolia (Baill.) Müll.Arg., Linnaea 34: 32. 1865. Acalypha filiformis var. ovalifo- 
lia (Baill.) Govaerts, World Checkl. Bibliogr. Euphorbiaceae 62. 2000. Type: Mayotte: Nov 1848. L. H. Boivin 3372 (holotype: P [P00196298]!).

Distribution. Comoros Archipelago (Anjouan, Mohéli, Mayotte).

References. Müller Argoviensis (1866: 852 as A. reticulata var. ovalifolia, 855); Baillon (1892: 1004); Palacký (1907: 25 as A. ovalifolia, 26); Voeltzkow (1917: 447); Pax and Hoffmann (1924: 127); Leandri (1942: 272); Govaerts et al. (2000: 62, 85, 105).

Notes. The specimens, indicated as isolectotypes, do not have Richard's original label. They instead have labels with Baillon's handwriting giving the location as "Madagascar". We believe that the correct location is the one indicated on the selected lectotype, "Ile Mohilla", whose current name is Mohéli, in the Comoros Archipelago. A search of specimens at P showed that Richard's collections numbered 284-286, 288, 290, 291 and 293 are also from Mohéli ( 289 has no locality and 292 is not listed). Richard 544 has an apparently original label showing the locality only as "Madagascar." Collections at $\mathrm{P}$ with nearby numbers are labelled as being from either "Nord de Madagascar" or "Ile Nos-bé" (now Nossi-bé), so this collection could be from northern Madagascar, although we have seen no other specimens from outside the Comoros Archipelago.

\section{Acalypha rottleroides Baill., Adansonia 1: 270. 1861.}

Type. Madagascar. Prov. Antsiranana: Nossibé, 1837, M. Richard 215 (holotype P [P00536728]!).

Acalypha polynema Baill., Hist. Pl. Madag., Atlas (1891) t. 187. Type: MadagasCAR: Plate 187 in Baillon ibid. loc., holotype.

Acalypha juliflora Pax, Bot. Jahrb. Syst. 19: 95. 1894. Type: Madagascar. Prov. Antsiranana: Nossibé, "Urwald von Loko-bé", Dec 1879, J. M. Hildebrandt 3279 (holotype: W [W1889-0089773]!; isotypes: JE [JE00004291]!, K [K000186527]!, P [P00536729!, P00536730!, P00536731!]).

Distribution. Madagascar (Antsiranana).

References. Müller Argoviensis (1866: 854); Baillon (1891: 182); Baillon (1895b: 1197); Baillon (1895b: 1197) A. polynema; Palacký (1907: 25) A. juliflora; Palacký (1907: 26) A. polynema; Palacký (1907: 26); Nitschke (1923: 280) A. juliflora; Pax and Hoffmann (1924: 127); Leandri (1942: 277); Govaerts et al. (2000: 70, 105) A. juliflora; Govaerts et al. (2000: 86).

\section{Acalypha spachiana Baill., Adansonia 1: 272. 1861.}

Acalypha spachiana var. latifolia Baill., Adansonia 1: 272. 1861.

Type. Madagascar. Prov. Antsiranana: Baies de Rigny et de Diego-Suarès, 1848, L. H. Boivin 2654 (lectotype, designated here: P [P00536733]!; isolectotype: G [G00034251]!, GDC [G00324359]!, P [P00536734]!). 
Acalypha spachiana var. acutifolia Baill., Adansonia 1: 272. 1861. Type: MadagasCAR. Prov. Antananarivo: Antananarivo, 12 Feb 1840, J. P. Goudot s.n. (holotype: G [G00383582]!, syn. nov.).

Acalypha spachiana var. minor Baill., Adansonia 1: 272. 1861. Type: MadAGASCAR: s.l., s.d., M. Bojer s.n. (holotype: P [P00536735]!; isotypes: TUB [TUB002081!, TUB002082]!, syn. nov.).

Acalypha buchenavii Müll.Arg., Abh. Naturwiss. Verein Bremen 7: 27. 1880.

Type: Madagascar. Prov. Antananarivo: Antananarivo, 18 Dec. 1877, D.C. Rutenberg s.n. (lectotype (probably holotype), designated here: BRNU [BRNU347926]!).

Acalypha squarrosa Pax, Bot. Jahrb. Syst. 19: 97. 1894. Type. Madagascar. Prov. Antananarivo: Antsirabe, "Sírabé", Aug 1880, J. M. Hildebrandt 3560 (holotype B or WRSL, presumably destroyed; lectotype, designated here: JE [JE00004308]!; isolectotype: JE [JE00004309]!, K [K000186510]!, P [P00536732]!).

Tragia saxatilis Bojer ex Pax \& K.Hoffm., Pflanzenr. 147, 16 (Heft 85): 33.1924 nom. nud.

Distribution. Madagascar (Antananarivo, Antsiranana, Mahajanga, Toamasina, Toliara).

References. Müller Argoviensis (1866: 827); Müller Argoviensis (1882: 27) A. buchenavii; Baillon (1892: 1003); Baillon (1895b: 1199) A. buchenavii; Palacký (1907: 26); Pax and Hoffmann (1924: 33); Pax and Hoffmann (1924: 33) A. squarrosa; Leandri (1942: 255, 257); Jenkins (1987: 347); Jenkins (1990: 408, 433); Govaerts et al. (2000: 89, 96); Govaerts et al. (2000: 89) A. squarrosa; Seebaluck et al. (2015: 153).

Notes. The holotype of Acalypha buchenavii should be at BREM but Rutenberg's specimens arrived at BRNU after World War II. Originally in the Überseemuseum in Bremen (BREM), they were transferred to northern Moravia (Czechia was at that time a Protectorate of Nazi Germany) to save them from potential destruction by bombardment. After the collapse of the Nazi regime, these collections were confiscated as "German property" and sent to BRNU.

\section{Acalypha urophylla Boivin ex Baill., Adansonia 1: 273. 1861.}

Acalypha reticulata var. urophylla (Boivin ex Baill.) Müll.Arg. Linnaea 34: 32. 1865. Acalypha filiformis var. urophylla (Boivin ex Baill.) Govaerts, World Checkl. Bibliogr. Euphorbiaceae 63. 2000.

Type. Madagascar. Prov. Antsiranana: Nossibé, plateau de Hell-Ville, Jun 1847, L. H. Boivin 2178 (lectotype, designated here: P [P00536752]!; isolectotypes: G [G00034246]!, GDC [G00324519]!, P [P00536751!, P00536753!]).

Syntypes. Madagascar: s.l., s.d., L. M. A. Du Petit-Thouars s.n. P [P00536748]!; Madagascar. Prov. Antsiranana: Nossibé, Dec 1840, A. Pervillé 364 P 
[P00536749]!; Madagascar. Prov. Antsiranana: Nossibé, M. Richard 385 P [P00536750]!.

Acalypha goudotiana Baill., Adansonia 1: 268. 1861. Acalypha reticulata var. goudotiana (Baill.) Müll.Arg., Linnaea 34: 32. 1865. Acalypha filiformis var. goudotiana (Baill.) Govaerts, World Checkl. Bibliogr. Euphorbiaceae 62. 2000. Type: MadAGASCAR: s.l., 1830, J. P. Goudot s.n. (holotype: G!; isotype: P [P00536727]! fragment), syn. nov.

Acalypha reticulata var. urophylla f. meeusei Leandri, Notul. Syst. (Paris) 10: 264. 1942. Type: Madagascar, Prov. Fianarantsoa, base Est du Pic d'Ivohibe, 19 Sep 1926, R. Decary 5352 P [P00224690]!; Prov. Toamasina, Analamazaotra forest, 1912, H. Perrier de la Bâthier 9741 (lectotype, designated here: P [ P05604377]!, syn. nov.). Syntypes. Madagascar, Prov. Toamasina, Soanierana, Antasibé, 9 Dec 1938, H. J. Lam \& A. D. J. Meeuse 5814 WAG [WAG0133229]!, L [L0242110]!; Moramanga, 900 m, 11 Nov 1938, H. J. Lam \& A. D. J. Meeuse 5363 L [L0242105]!; Analamazaotra, 1000 m, 10 Nov. 1938, H. J. Lam \& A. D. J. Meeuse 5290 L [L0242106]!.

Acalypha reticulata var. urophylla f. longa Leandri, Notul. Syst. (Paris) 10: 265. 1942. Type: Madagascar, Prov. Toliara, bassin de la Manampanihy, Col de Fitana, 700 m, 15 Oct 1928, H. Humbert 6015 (lectotype, designated here: P [P05604383]!, syn. nov.).

Syntypes. Madagascar, Prov. Toliara, Col d'Ivolo (District de Fort-Dauphin), 500 m, 5 Sept 1932, R. Decary 10558 P [P05604384]!; Fort-Dauphin, col de Tsitongabarika, 600 m, 9 Sept 1932, R. Decary 10595 P [P05604399]!.

Acalypha reticulata var. urophylla f. vohitrae Leandri, Notul. Syst. (Paris) 10: 265. 1942. Type: Madagascar, Prov. Toamasina, Andevorante, rive droite de la Vohitra près de Lohariandava, 200-250 m, 10 Oct 1912, R. Viguier \& H. Humbert 661 (lectotype, designated here: P [P05604437]!; isolectotypes: P [P05604440!, P05604441!], syn. nov.).

Acalypha reticulata var. urophylla f. glabrescens Leandri, Notul. Syst. (Paris) 10: 266. 1942. Type: Madagascar, Prov. Antsiranana, Massif du Tsaratanana, 2200 m, H. Perrier de la Bâthie 16180 (lectotype, designated here: P [P05604386]! syn. nov.). Syntypes. Madagascar, Prov. Antsiranana, Sambirano, Nov-Dec 1937, H. Humbert 18659 P [P05604397!, P05604400!]; Massif du Tsaratanana, Sep 1912, H. Perrier de la Bâthie 18614 P [P05604382]!; Massif du Tsaratanana, 1600 m, H. Perrier de la Bâthie 15371 P [P05604385]!; MadAgascar, Prov. Fianarantsoa, Ranohira, Isalo, 30 Jul 1928, H. Humbert 5014 P [P05604401!, P05604402!]; Madagascar, Prov. Mahajanga, Beritsoka, H. Perrier de la Bâthie $413 \mathrm{P}$ [P05604392!, P05604390!]; Beritsoka, Dec 1897, H. Perrier de la Bâthie 422 P [P05604387!, P05604388!, P05604391!]; Beritsoka, H. Perrier de la Bâthie 9822 P [P05604389]!; Madagascar, s. 1., R. Baron 5987 P [P05604407]!; s. 1., L. Humblot 335 P [P05604393!, P05604394!, P05604395!, P05604396!].

Acalypha reticulata var. urophylla f. typique Leandri, Notul. Syst. (Paris) 10: 262. 1942 nom. inval. 
Distribution. Madagascar (Antananarivo, Antsiranana, Fianarantsoa, Mahajanga, Toamasina and Toliara). Comoros Archipelago (Grande Comore, Anjouan, Mohéli and Mayotte).

References. Müller Argoviensis (1866: 260, 852) as A. reticulata var. goudotiana and $A$. reticulata var. urophylla; Baron (1889: 262); Baillon (1891: 189); Baillon (1892: 1004); De Candolle (1901: 567); Palacký (1907: 25 as A. goudotiana, 26); Pax and Hoffmann (1924: 105, 260) as as $A$. reticulata var. goudotiana and $A$. reticulata var. urophylla; Leandri (1942: 258, 260, 262) as A. reticulata var. goudotiana and $A$. reticulata var. urophylla; Leandri (1942: 281) as A. emirnensis; Leandri (1948: 186) as $A$. reticulata var. goudotiand; Govaerts et al. (2000: 62, 63, 100, 105, 108).

Notes. Acalypha urophylla has been considered to be a variety of $A$. reticulata, but A. urophylla can be distinguished by its sessile female bracts with dentate margins vs. pedicellate female bracts with crenate to subentire margins in $A$. filiformis. (see notes in A. filiformis). See notes under $A$. gracilipes, $A$. lamiana and $A$. paxii for the differences between those species and $A$. urophylla.

\section{Acalypha vulneraria Baill., Bull. Mens. Soc. Linn. Paris 2: 1180-1181. 1895.}

Type. Madagascar. Prov. Toliara: Fort-Dauphin, s.d., G. F. Scott-Elliot 3010 (lectotype, designated here: P [P00536740]!; isolectotypes: K [K000186511]!, P [P00536739]!).

Syntypes. Madagascar. Prov. Toliara: Fort-Dauphin, M. Cloisel 51 (wrongly transcribed as "Cloisel 50" in the protologue) P [P04779526]!.

Distribution. Madagascar (Fianarantsoa, Mahajanga, Toliara).

References. Palacký (1907: 26); Nitschke (1923: 281); Pax and Hoffmann (1924: 128); Leandri (1935: 46); Leandri (1942: 279); Govaerts et al. (2000: 93).

\section{Acalypha wilkesiana Müll.Arg., Prodr. 15(2): 817. 1866.}

Ricinocarpus wilkesianus (Müll.Arg.) Kuntze, Revis. Gen. Pl. 2: 618. 1891. Acalypha amentacea Roxb. subsp. wilkesiana (Müll.Arg.) Fosberg, Smithsonian Contr. Bot. 45: 10.1980.

Type. Fiji, s.d., (U.S. Expl. Exped. Under. Capt. Wilkes), B. C. Seeman s.n. (holotype: G-DC [G00324022]!; isotypes: GH [00045512]!, K [K000959008]!, US [00096423!, $00096424 !])$.

Distribution. Tropical Africa. Madagascar (Antananarivo).

References. Robertson (1989: 200).

Notes. Shrub native to Fiji (Melanesia), used as an ornamental plant throughout the tropics. It has been reported from Seychelles (Robertson 1989). We found some specimens from Madagascar 


\section{Doubtful or excluded species}

Acalypha neptunica Müll.Arg., Abh. Naturwiss. Vereins Bremen 7: 26. 1880. Type. TanZania, Zanzibar: Kidosi, Oct 1873, J. M. Hildebrandt 1146. (lectotype, designated by Cardiel and Montero Muñoz (2018): K [K000431078]!; isolectotype: G [G00007675]!).

This species occurs in West Tropical Africa, West Central Tropical Africa, Northeast Tropical Africa and East Tropical Africa. There are only two references of Acalypha neptunica from Madagascar. They appear in Baillon (1895b) and in the checklist of Palacký (1907). We have not yet confirmed the presence of this species in the study area.

Acalypha spiciflora Burm.f., Fl. Ind. 203, pl. 61-2. 1768. Claoxylon spiciflorum (Burm.f.) A.Juss., Euphorb. Gen. 43. 1824. Cleidion spiciflorum (Burm.f.) Merr., Interpr. Herb. Amboin. 322. 1917.

Acalypha spiciflora Burm.f. was described and illustrated in Burman's Flora Indica, but the plate and the description are very imprecise. Subsequently, Poiret (1804) cited under this name a specimen in the Lamarck herbarium, P00382113, from Réunion. That specimen is morphologically close to Burman's plate, but it is not an Acalypha species. Later, Jussieu combined A. spiciflora under the genus Claoxylon, as Cla. spiciflorum. Merrill combined it under Cleidion as Cle. spiciflorum, the name that is accepted today. Müller Argoviensis (1866) wrongly placed A. spiciflora as a synonym of $A$. acuminata Vahl ex Baill. Pax and Hoffmann (1924) and Leandri (1942) followed the treatment of Müller Argoviensis, but, applying the rule of priority, chose $A$. spiciflora as the accepted name. This is the origin of the confusion in the use of this name.

Acalypha venosa Poir., Encycl. 6: 204. 1804.

The type specimen of Acalypha venosa (Commerson s.n., from Madagascar) corresponds to Leptonema venosum (Poir.) A.Juss. (Phyllanthaceae), as was pointed out by Steudel (1841: 31).

\section{Acknowledgements}

This study was partly funded by the Universidad Autónoma de Madrid (Spain). Also, the project has received support from SYNTHESYS Project (http://www.synthesys. info/; FRTAF6307) which is financed by European Community Research Infrastructure Action under the FP7 "Capacities" Programme. Levin's research was partially funded by a grant from the United States National Science Foundation, DEB0128872. We kindly thank the curators and staff of the aforementioned herbaria for facilitating the study of their specimens, especially thanks to Marc Jeanson, Grégoire Flament, Cécile Aupic and Virginie Bouetel from P herbarium and Nicolas Fumeaux from $\mathrm{G}$ for helping the first author during her stay at P and G. Finally, our gratitude to the editor and reviewers for their suggestions and helpful comments. 


\section{References}

Baillon HE (1858) Etude générale du groupe des Euphorbiacées. Librarie de Victor Masson, Paris, 1-700. https:// doi.org/10.5962/bhl.title.50439

Baillon HE (1861) Euphorbiacées Africaines. Deixiéme partie, Afrique Orientale (Bourbon, Maurice, Madagascar, Zanguebar, etc.). Adansonia 1: 251-286.

Baillon HE (1891) Histoire Naturelle des Plantes Vol. 34, Tome 4 (Atlas 2), $3^{\text {a }}$ partie, 27 fascicule. In: Grandidier A. (ed.) Histoire Physique, Naturelle et Politique de Madagascar. M. le Grade des Sceaux, à l'Imprimerie Nationale, Paris, 161-204.

Baillon HE (1892) Liste des plantes de Madagascar (suite de la page 996). Bulletin Mensuel de la Société Linnéenne de Paris 2(126): 1003-1005.

Baillon HE (1895a) Liste des plantes de Madagascar (suite de la page 1005). Bulletin Mensuel de la Société Linnéenne de Paris 2(149): 1177-1184.

Baillon HE (1895b) Liste des plantes de Madagascar (suite de la page 1181). Bulletin Mensuel de la Société Linnéenne de Paris 2(151): 1193-1200.

Baker JG (1883) Contributions to the Flora of Madagascar. Part III. Incompletae, Monocotyledons, and Filices. Journal of the Linnean Society. Botany 20: 237-304.

Baker JG (1884) Further Contributions to the Flora of Madagascar. Second and Final Part. Journal of the Linnean Society. Botany 21: 407-455.

Baker JG (1877) Flora of Mauritius and the Seychelles: a description of the flowering plants and ferns of those islands. L Reeve, London, 1-557.

Baron R (1889) The Flora of Madagascar. Journal of the Linnean Society. Botany 25: 246-294. Berry PE, Kainulainen K, van Ee BW (2017) A Nomenclator of Croton (Euphorbiaceae) in Madagascar, the Comoros Archipelago, and the Mascarene Islands. PhytoKeys 90: 1-87. https://doi.org/10.3897/phytokeys.90.20586

Bojer W (1837) Hortus Mauritianus. Aimé Mararot \& Co., Mauritius, 1-456.

Brummitt RK (2001) World geographical scheme for recording plant distributions, 2nd ed. Plant Taxonomic Database Standards, No. 2. International Working Group on Taxonomic Databases for Plant Sciences (TDWG). Hunt Institute for Botanical Documentation Carnegie Mellon University, Pittsburgh, 1-137.

Cardiel JM, Montero Muñoz I (2018) Synopsis of Acalypha (Euphorbiaceae) of West Tropical Africa, including Cameroon, Chad, Equatorial Guinea, Gabon, and São Tomé and Príncipe Plant Systematics and Evolution 304: 93-110. https://doi.org/10.1007/s0060

Cardiel JM, Muñoz-Rodríguez P, Dorda E, Pardo de Santayana M (2018) Acalypha Taxonomic Information System. http://www.acalypha.es [accessed 03.05.2018]

Coode MJE (1979) Notes on Euphorbiaceae in the Mascarene Islands: II. Kew Bulletin 34(1): 39-48. https://doi.org/10.2307/4117969

Coode MJE (1982) Acalypha L. In: Bosser J, Cadet T, Guého J, Marais W (Eds) Flore des Mascareignes: La Réunion, Maurice, Rodrigues. 153. Lauracées à 160. Euphorbiacées. The Sugar Industry Research Institute, Mauritius, L'Office de la Recherche Scientifique et Technique Outre-Mer, Paris, and The Royal Botanic Gardens, Kew, 68-79.

de Cordemoy EJ (1895) Flore de l'île de la Réunion (phanérogames, cryptogames, vasculaires, muscinées) avec l'indication des propriétés économiques \& industrielles des plantes. Jacques Lechevalier, Librairie Médicale \& Scientifique, Paris, 1-574. 
De Candolle A (1901) Plantae Madagascarienses ab Alberto Mocquerysio lectae. Bulletin de l'Herbier Boissier, sér. 2 1(6): 564-567.

Fosberg FR (1974) Miscellaneous notes on the flora of Aldabra and neighbouring islands: III*. Kew Bulletin 29(2): 253-266. https://doi.org/10.2307/4108539

Goodman SM (1996) A Floral and faunal inventory of the eastern slopes of the Réserve naturelle intégrale d'Andringitra, Madagascar: with reference to elevational variation. Fieldiana, Zoology, new. ser. 85: 1-319.

Govaerts R, Frodin DG, Radcliffe-Smith A (2000) World Checklist and Bibliography of Euphorbiaceae (and Pandaceae). Royal Botanic Gardens, Kew, 1-1621.

Heckel E (1903) Catalogue alphabetique raisonne des plantes medicales et toxiques de Madagascar avec leur emploi indigene. Annales du Museé colonial de Marseille v. 11 (s. 2, v. 1), fasc. 2: 59-204.

Hemsley WB (1919) Flora of Aldabra: With Notes on the Flora of the Neighbouring Islands. Bulletin of Miscellaneous Information (Royal Botanic Gardens, Kew) 3: 108-153.

Horn JW, van Ee BW, Morawetz JJ, Riina R, Steinmann VW, Berry PE, Wurdack KJ (2012) Phylogenetics and the evolution of major structural characters in the giant genus Euphorbia L. (Euphorbiaceae). Molecular Phylogenetics and Evolution 63(2): 305-326. https://doi. org/10.1016/j.ympev.2011.12.022

Hutchinson J (1913) Acalypha. In: Thiselton-Dyer WT (Ed.) Flora of tropical Africa 6, 1: nyctagineae-euphorbiaceae. Lovell Reeve \& Co., London, 880-910.

Jenkins MD (1987) Madagascar, an environmental profile. IUCN, Gland, Switzerland and Cambridge, U.K., 1-396.

Jenkins MD (1990) Madagascar: profil de l'environnement. IUCN, Gland, Switzerland and Cambridge, U.K., 1-464.

Koehne E (1892) Just's botanischer jahresbericht. Systematisch geordnetes repertorium der botanischen literatur aller länder, Jahrg. 20, Abt. 1. Gebr. Borntraeger, Leipzig, Berlin, $1-636$.

Leandri J (1935) Catalogue des Plantes de Madagascar, Euphorbiaceae. Pitot G, Tananarive, 1-51. Leandri J (1942) Acalypha. Contribution a l'etude des Euphorbiacées de Madagascar (VI). Notululae Systematicae 10: 252-290.

Leandri J (1948) Euphorbiacées récoltées a Madagascar et aux Mascareignes par MM. Lam et Meeuse en 1938. Notulae Systematicae 13: 182-187.

Leandri J (1952) Les arbres et grands arbustes malgaches de la familie Euphorbiacées. Naturaliste Malgache 4(1): 47-82.

Levin GA, Steinmann VW, Sagun VG (2005) Phylogeny and biogeography of Acalypha (Euphorbiaceae). Abstracts of the XVII International Botanical Congress: 68. Vienna, Austria.

McNeill J, Barrie FR, Buck WR, Demoulin V, Greuter W, Hawksworth DL, Herendeen PS, Knapp S, Marhold K, Prado J, Prud'homme Van Reine WF, Smith GF, Wiersema JH, Turland NJ (Eds) (2012) International Code of Nomenclature for algae, fungi, and plants (Melbourne Code) adopted by the Eighteenth International Botanical Congress Melbourne, Australia, July 2011. Regnum Vegetabile 154. Koelz Scientific Books.

Müller-Argoviensis J (1865) Euphorbiaceae. Vorläufige Mittheilungen aus den für De Candolle Prodromus bestimmtem Manuscript über diese Familie. Linnaea 34: 1-54. 
Müller-Argoviensis J (1866) Acalypha. In: De Candolle AP (Ed.) Prodromus Systematis Naturalis Regni Vegetabilis. Vol 15(2). Paris, Treuttel et Würtz, 799-889.

Müller-Argoviensis J (1882) Euphorbiaceen. In: Buchenau, Fr (Ed.) Reliquiae Rutenbergianae I (Botanik, Taf. I, II). Abhandlungen herausgegeben vom Naturwissenschaftlichen Verein zu Bremen 7: 24-30.

Nitschke R (1923) Die geographische Verbreitung der Gatung Acalypha. In: Mez K (Ed.) Botanisches Archiv: Zeitschrift für die gesamte Botanik. Verlag des Repertoriums, Berlin, 277-317.

Palacký J (1907) Catalogus plantarum Madagascariensium II. Pragae, 1-89.

Pax FA (1890) Euphorbiaceae. In: Engler A, Prantl K (Eds) Die natürlichen Pflanzenfamilien 3(5): $1-123$.

Pax FA (1894) Enphorbiaceae africanae. II. In: Engler A (Ed.) Beiträge zur Flora von Afrika. VIII. Botanische Jahrbücher fur Systematik, Pflanzengeschichte und Pflanzengeographie 19: 76-127.

Pax FA, Hoffmann K (1924) Acalypha. In: Engler A (Ed.) Das Pflanzenreich. IV, 147-16 (heft 85), 1-231.

Poiret JLM (1804) Acalypha. In: Lamarck L, Poiret JLM (Eds) Encyclopédie Métodique. Botanique. Paris, 6: 202-209.

Renvoize SA (1975) A Floristic Analysis of the Western Indian Ocean Coral Islands. Kew Bulletin 30(1): 133-152. https://doi.org/10.2307/4102881

Radcliffe-Smith A (1987) Euphorbiaceae (Part 1). In: Polhill RM (Ed.) Flora of tropical East Africa. Balkema, Rotterdam, 185-213.

Radcliffe-Smith A (1990) New record of Acalypha in Australia. Kew Bulletin 45(4): 677-679. https://doi.org/10.2307/4113874

Radcliffe-Smith A (1996) Euphorbiaceae. In: Pope GV (Ed.) Flora Zambesiaca, vol 9. Part 4. Royal Botanic Gardens, Kew, 1-138.

Robertson SA (1989) Flowering Plants of Seychelles. Royal Botanic Gardens, Kew, 1-343.

Schatz GE (2001) Generic tree flora of Madagascar. Royal Botanic Gardens, Kew, Richmond, United Kingdom, 1-477.

Seebaluck R, Gurib-Fakim A, Mahomoodally F (2015) Medicinal plants from the genus Acalypha (Euphorbiaceae)-A review of their ethnopharmacology and phytochemistry. Journal of Ethnopharmacology 159: 137-157. https://doi.org/10.1016/j.jep.2014.10.040

Steudel ET (1841) Nomenclator Botanicus seu: synonymia plantarum universalis, enumerans ordine alphabetico nomina atque synonyma, tum generic tum specifica et A Linnaeo et A recentioribus de re botanica scriptoribus plantis phanerogamis imposita. Pars II. Stuttgartiae et Tubingae, Typis et Sumptibus, Cottae, 1-810.

Thiers B (2018) Index Herbariorum: a global directory of public herbaria and associated staff. New York Botanical Garden's Virtual Herbarium. http://sweetgum.nybg.org/ih/ [accessed 03.05.2018]

Voeltzkow A (1917) Flora und Fauna der Comoren. In: Voetzkow A (Ed.) Reise in Ostafrika in den Jahren 1903-1905. Wissenschaftliche Ergebnisse, Stuttgart, Schweizerbart 3: 429-480. 